\title{
TRPA1-expressing lamina propria mesenchymal cells regulate colonic motility
}

\author{
Yanjing Yang, ${ }^{1,2,3}$ Shenglan Wang, ${ }^{1,2,4}$ Kimiko Kobayashi, ${ }^{3}$ Yongbiao Hao, ${ }^{1,5}$ Hirosato Kanda, ${ }^{1,2,3}$ \\ Takashi Kondo, ${ }^{5}$ Yoko Kogure, ${ }^{1}$ Hiroki Yamanaka, ${ }^{3}$ Satoshi Yamamoto, ${ }^{1}$ Junxiang Li, ${ }^{6}$ Hiroto Miwa, ${ }^{2,5}$ \\ Koichi Noguchi, ${ }^{3}$ and Yi Dai ${ }^{1,2,3}$ \\ 'Department of Pharmacy, School of Pharmacy, Hyogo University of Health Sciences (HUHS), Kobe, Hyogo, Japan. \\ ${ }^{2}$ Traditional Medicine Research Center, Chinese Medicine Confucius Institute at Hyogo College of Medicine (CMCIHCM), \\ Kobe, Hyogo, Japan. ${ }^{3}$ Department of Anatomy and Neuroscience, Hyogo College of Medicine (HCM), Nishinomiya, \\ Hyogo, Japan. ${ }^{4}$ School of Acupuncture-Moxibustion and Tuina, Beijing University of Chinese Medicine (BUCM), Beijing, \\ China. ${ }^{5}$ Division of Gastroenterology, Department of Internal Medicine, HCM, Nishinomiya, Hyogo, Japan. ${ }^{6}$ Division of \\ Gastroenterology, Department of Internal Medicine, Dongfang Hospital of BUCM, Beijing, China.
}

The physiological process of defecation is directly controlled by colorectal motility. The transient receptor potential ankyrin 1 (TRPA1) channel is expressed in small intestine enterochromaffin cells and is involved in gastrointestinal motility via serotonin release. In the colorectum, however, enterochromaffin cell localization is largely distinct from that in the small intestine. Here, we investigated the role of lower gastrointestinal tract TRPA1 in modulating colorectal motility. We found that in colonic tissue, TRPA1 is predominantly expressed in mesenchymal cells of the lamina propria, which are clearly distinct from those in the small intestine. These cells coexpressed COX1 and microsomal prostaglandin E synthase-1. Intracolonic administration of TRPA1 agonists induced colonic contraction, which was suppressed by a prostaglandin E2 (PCE2) receptor 1 antagonist. TRPA1 activation induced calcium influx and PGE2 release from cultured human fibroblastic cells. In dextran sulfate sodium-treated animals, both TRPA1 and its endogenous agonist were dramatically increased in the colonic lamina propria, accompanied by abnormal colorectal contractions. Abnormal colorectal contractions were significantly prevented by pharmacological and genetic inhibition of TRPA1. In conclusion, in the lower gastrointestinal tract, mesenchymal TRPA1 activation results in PCE2 release and consequently promotes colorectal contraction, representing what we believe is a novel physiological and inflammatory bowel disease-associated mechanism of gastrointestinal motility.

Authorship note: YY and SW contributed equally to this work.

Conflict of interest: The authors have declared that no conflict of interest exists.

Copyright: (c) 2019 American Society for Clinical Investigation

Submitted: May 22, 2018

Accepted: April 2, 2019

Published: May 2, 2019.

Reference information: JCl Insight. 2019;4(9):e122402. https://doi. org/10.1172/jci.insight.122402.

\section{Introduction}

Bowel movement is one of the most important physiological activities in humans. Both nutrient absorption and waste removal depend on normal bowel motility. Abnormal bowel movements comprise the major symptom of various intestinal disorders and are typically accompanied by constipation or diarrhea. Normal colonic movement is achieved through an integrated process including coordinated smooth muscle contraction and relaxation, autonomous nervous system regulation, and enteric nervous system involvement, which occur concurrently with the release of multiple neurohumoral substances (1). Many neuromodulators or neurotransmitters such as calcitonin gene-regulated peptide (CGRP), substance P (SP), serotonin (5-hydroxytryptamine, or 5-HT), and prostaglandin E2 (PGE2) contribute to the modulation of bowel movement (1).

Transient receptor potential ankyrin 1 (TRPA1) is a nonselective cation channel that is expressed in sensory neurons and other cell types (2). TRPA1 is activated by low temperature $\left(\leq 18^{\circ} \mathrm{C}\right)$, osmotic stimuli (3), and many natural plant-derived products such as garlic, mustard, and cinnamon (4), which are often consumed as ingredients in condiments. TRPA1 is also activated by endogenous metabolites of oxidative stress including 4-oxo-2-nonenal and 4-hydroxy-2-nonenal (4-HNE) $(5,6)$. In addition to its role as a pain sensor, accumulating evidence indicates that TRPA1 has functions aside from the somatosensory system (7). 
TRPA1 channels are reportedly expressed on several non-neuronal cells (8-10). Recent studies have indicated that TRPA1 channels are expressed by enterochromaffin (EC) cells and that their activation can regulate small intestinal motility through 5 -HT release $(10,11)$. The role of TRPA1 in the regulation of gastrointestinal (GI) motility was thus considered to be dependent on EC cell function. However, heterogeneity and regional differences of EC cells along the GI tract has been explored by recent studies using gene expression profiling $(12,13)$. This finding led us to question whether there are regional differences of 5-HT and TRPA1 expression in EC cells along the GI tract and with what consequences for the different modulation mechanisms of GI motility.

In the present study, we identified predominant expression of TRPA1 in mesenchymal cells (and not in EC cells) of the colon. The TRPA1-expressing colonic mesenchymal cells were found to produce COX1 and microsomal prostaglandin E synthase-1, of which the downstream product PGE2 acts as a modulator of colonic motility. We then investigated the role and mechanism of these TRPA1-expressing cells in modulating colonic motility in both normal and inflammatory bowel disease (IBD) conditions. Our data demonstrated a previously unknown mechanism underlying the modulation of large intestinal movement, which involves TRPA1-expressing mesenchymal cells in the colonic lamina propria.

\section{Results}

TRPA1 agonists increase colorectal contraction in naive rats. We designed an experimental system to investigate the role of TRPA1 channels in colorectal motility in vivo (Supplemental Figure 1A; supplemental material available online with this article: https://doi.org/10.1172/jci.insight.122402DS1). For the recording system, the base pressure was set at $10 \mathrm{mmHg}$ to observe colonic motility. A rhythmic movement with a short interval (relaxation) and low peak value (contraction) was recorded in naive rats. This contraction value was always less than $20 \mathrm{mmHg}$ and did not evoke a pain response in naive rats (Figure 1 and Supplemental Figure 1B). Thus, traces that were greater than $20 \mathrm{mmHg}$ were considered enhanced contraction. Vehicle application did not change the contraction value, which was maintained below $20 \mathrm{mmHg}$. The rapid application of 2 TRPA1 agonists, allyl isothiocyanate (AITC) and cinnamaldehyde (within 3 minutes) induced irregular and violent contractions (Figure 1, A, C, and D). In contrast, a transient receptor potential cation channel subfamily V member 1 (TRPV1) agonist, capsaicin, did not have a distinct effect on colorectal contraction, although a slight increase was recorded in the high-concentration group (Figure 1, B and E). The accumulating contraction values were increased in a dose-dependent manner by AITC treatment $(1050.4 \pm 396.1 \mathrm{mmHg} \cdot \mathrm{s}$ for $0.3 \mathrm{mM}, 1509.1$ $\pm 189.2 \mathrm{mmHg} \cdot \mathrm{s}$ for $1 \mathrm{mM}$, and $2149.8 \pm 864.6 \mathrm{mmHg} \cdot \mathrm{s}$ for $3 \mathrm{mM}$ AITC) compared with those in the vehicle group, as well as pretreatment levels (Figure 1C and Supplemental Figure 1C). The contractions intermittently occurred for accumulated times of 89, 164, and 229 seconds within a 15-minute recording time following AITC treatment at $0.3,1$, or $3 \mathrm{mM}$, respectively (Supplemental Figure 1D). Both A967079 and HC030031, TRPA1 selective antagonists significantly suppressed TRPA1 agonist-induced increases in colorectal contraction (Figure 1, A, C, and D). No significant pain response was recorded in our system after AITC or capsaicin administration (Supplemental Figure 1E). Thus, extrinsic neuronal TRPA1 or TRPV1 might not be involved during AITC or capsaicin intracolonic responses, respectively, under the current conditions. As a positive control of the pain response recording system, we used colorectal distention with our current balloon apparatus to induce a pain response and recorded the electromyography from external oblique musculature in WT mice and Trpa1-KO mice. A significant increased pain response after colorectal distention at $60 \mathrm{mmHg}$ was found in WT mice compared with Trpa1-KO mice (Supplemental Figure 7), which was consistent with the previous report (14), suggesting the balloon apparatus used in the present experiments is accurate and reliable. These results suggest that activation of TRPA1 (but not TRPV1) in the local colonic tissue induces bowel contractions.

TRPA1 is expressed abundantly in the vimentin-positive cells of the lamina propria in the colonic mucosa. A recent study indicated that TRPA1 is expressed by EC cells in the small intestine and that its activation modulates gut motility by increasing 5-HT release (10). To determine if the observed TRPA1 agonist-induced increase in colonic contraction (Figure 1) involves EC cells, we investigated the expression of Trpa1 and its co-localization with 5-HT in the rat GI tract. We observed that Trpa1 mRNA was clearly localized to 5-HT-immunoreactive EC cells in the small intestine, in accordance with the previous study (10). However, Trpa1 mRNA-labeled cells in the colon were mainly localized to the apical side of the lamina propria (Figure $2 \mathrm{~A}$ ), and only $3.18 \% \pm 0.81 \%$ of these cells coexpressed 5 -HT (Figure $2 \mathrm{~B}$ ). We noticed 
that the number of 5 -HT-containing cells in the colon $(0.37 \pm 0.12 /$ crypt $)$ was significantly less than that in the small intestine $(0.91 \pm 0.15$ /crypt) (Figure $2, \mathrm{~B}, \mathrm{C}$ and F). These 5 -HT-containing cells in the colon were mainly located in the crypts and subepithelium, whereas in the jejunum, these cells were found in the crypts and epithelium of villi (Figure 2, B and C). $87.24 \% \pm 9.27 \%$ of the total Trpa1 mRNA-labeled cells co-stained with 5-HT in the epithelial layer of the small intestine, which was significantly higher than that in the colon (Figure 2, G and H). To further identify Trpa1-positive cell types in the colon, we performed double staining for Trpal mRNA and the common mesenchymal cell marker, vimentin. We found that a predominant population of Trpa1-positive cells $(88.65 \% \pm 1.42 \%)$ showed vimentin immunoreactivity in the lamina propria (Figure 2D). In contrast, vimentin-positive cells rarely coexpressed Trpal in the small intestine (the number of Trpa1 mRNA-leveled cells was too small in the lamina propria to count for statistics) (Figure 2E). After confirming the specificity of the TRPA1 antibody using peptide antigens (Supplemental Figure 2A), double immunostaining was performed for vimentin (or 5-HT) and TRPA1. Consistent with the in situ hybridization studies, TRPA1 was mainly expressed in mesenchymal cells within the lamina propria of the colon (Supplemental Figure 2, B and C). To further identify the cell type of these TRPA1-positive mesenchymal cells in the lamina propria, we performed double immunostaining of TRPA 1 with $\alpha$-smooth muscle actin ([ $\alpha$-SMA] a myofibroblast marker), lymphocyte common antigen ([CD45] a leukocyte marker), or CD31 (an endothelial cell marker). $\alpha$-SMA, CD45, or CD31 was expressed at low levels in the apical area of the lamina propria in naive rats and rarely coexpressed with TRPA1 in the lamina propria (Supplemental Figure 2, D-F). Moreover, we performed double immunostaining using human tissues from healthy volunteers to characterize TRPA1 expression and found that it was also coexpressed with vimentin in the human colorectal mucosa (Supplemental Figure 3).

Among the different modulators of intestinal motility, endogenous PGE2 plays an essential role in regulating longitudinal and circular muscle motility (15-17).

We thus confirmed that i.p. injection of PGE2 produced obvious colorectal contractions in naive rats (1531.7 $\pm 431.4 \mathrm{mmHg} \cdot \mathrm{s}$ based on the AUC value; Supplemental Figure 4). Because histological data did not indicate that 5-HT is a major player in TRPA1-mediated colorectal motility, we sought to determine whether PEG2 is involved in this event. We found that both Cox1 mRNA-labeled and COX1-immunoreactive cells in the lamina propria were vimentin positive (Figure 3, A, B, and E). In addition to COX1, the prostaglandin synthase $m P g e s 1$ was also expressed in vimentin-immunopositive cells of the colonic lamina propria (Figure 3, C and D). In addition, TRPA1-immunopositive cells in the lamina propria exhibited COX1 immunoreactivity (Figure 3F). In contrast, the expression of other neuromodulators or neurotransmitters such as COX2, 5-HT, SP, or CGRP was low in the colonic lamina propria of naive rats and was rarely coexpressed with vimentin (Supplemental Figure 5). These results suggested a potential function for colonic mesenchymal cells in releasing PGE2 following TRPA1 activation.

The human colonic fibroblast cell line expresses TRPA1 and releases PGE2 following AITC treatment. To further verify the functional interaction between TRPA1 and PGE2 in fibroblasts, we used the human colonic fibroblast cell line (CCD-18Co; American Type Culture Collection) and measured PGE2 release after AITC stimulation. First, we verified that TRPA1 is abundantly expressed in CCD-18Co cells (Figure 4, A and $\mathrm{B})$ and that AITC $(0.1 \mathrm{mM})$ obviously induces an intracellular calcium increase, which was inhibited by HC030031 (10 $\mu \mathrm{M}$; Figure $4 \mathrm{C})$. The $\mathrm{EC}_{50}$ value for AITC- or cinnamaldehyde-induced calcium influx was $34.4 \mu \mathrm{M}$ or $216.1 \mu \mathrm{M}$, respectively (Supplemental Figure 6, A and B). In contrast, neither expression nor activation of TRPV1 was observed in CCD-18Co cells (Figure 4, A and B and Supplemental Figure $6 \mathrm{C}$ ), which was consistent with our in vivo data. Next, we determined if TRPA1 activation could induce PGE2 release from fibroblasts. We found that AITC treatment for 3 minutes induced obvious PGE2 release in a dose-dependent manner, which was inhibited by A967079 (10 $\mu \mathrm{M}$ : Figure 4D). These data, in addition to in vivo data, have proved that TRPA1 is expressed by colonic fibroblasts and that its functional activation results in intracellular calcium influx and PGE2 release in these cells.

AITC-induced colorectal contraction is dependent on EP1 receptor activation. PGE2 can bind 4 subtype receptors in the GI tract to modulate intestinal motility and secretion (16). To determine the targeted receptor for TRPA1 activation-induced PGE2 in the colon, we examined the effects of i.p. injection of PGE2 receptor antagonists on AITC-induced colonic contraction. We found that both the EP1/2 and EP1 antagonists clearly inhibited AITC-induced colonic contraction, whereas EP3 and EP4 antagonists showed no inhibitory effects (Figure 5, A and B). Considering that EP2 contributes to relaxation but not contraction (18), and based on the aforementioned pharmacological and histological data, these results suggest that TRPA1 
A

Motility

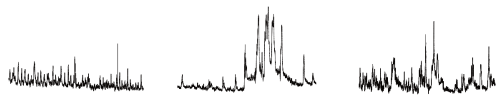

Electromyography

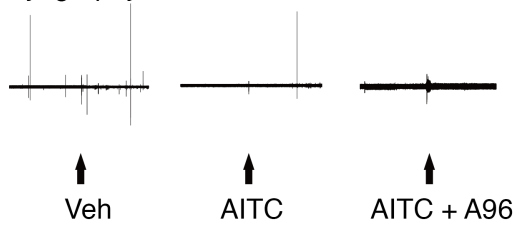

B

Motility

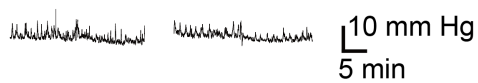

Electromyography

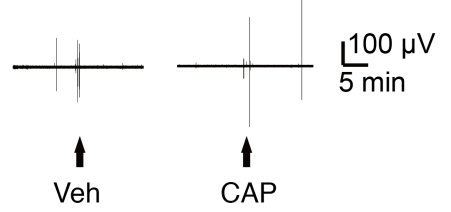

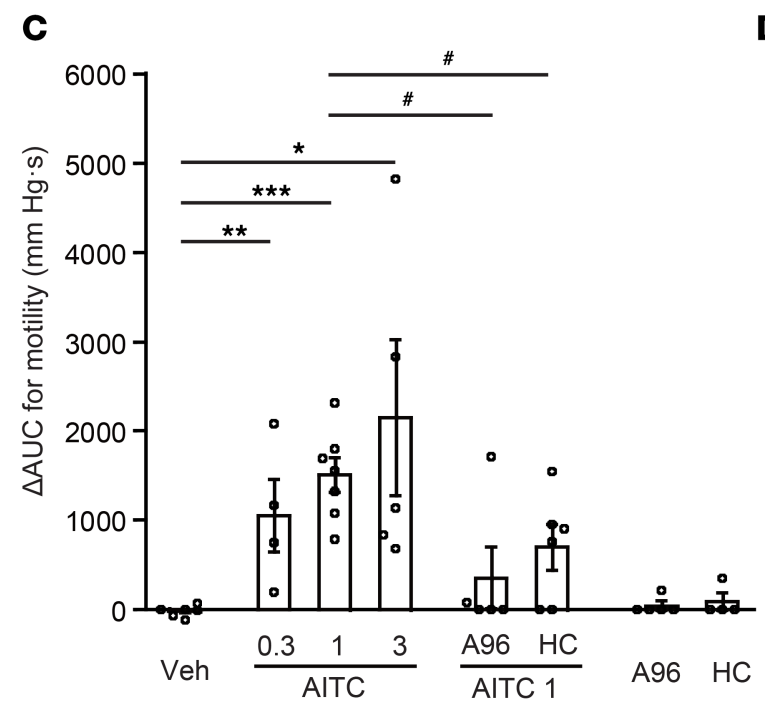
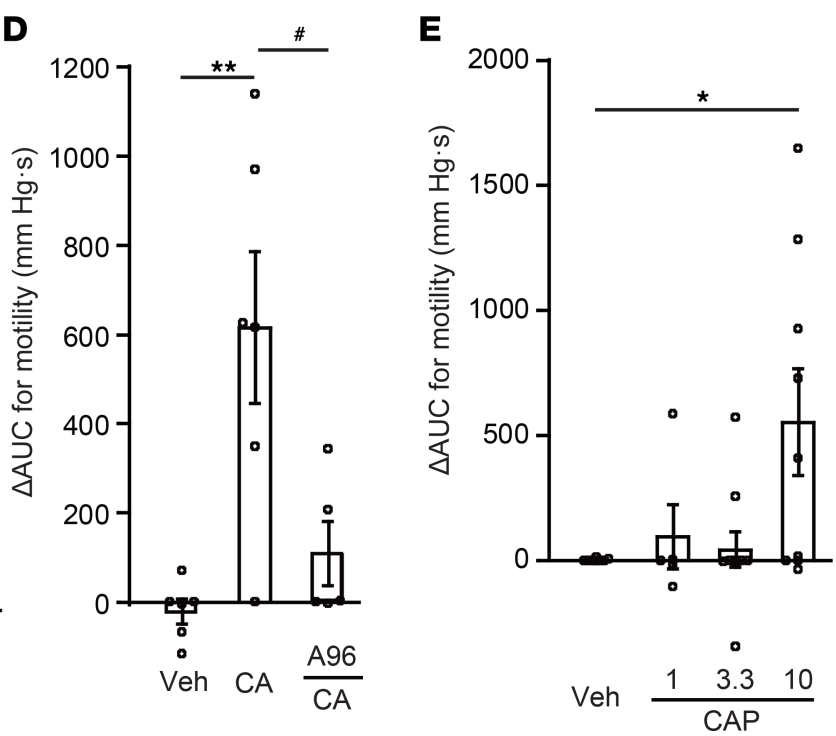

Figure 1. TRPA1 agonists increase colorectal contraction in rats. All agonists or antagonists were intracolonically administered. Saline (0.5 ml) with $0.02 \%$ DMSO was used as a vehicle (Veh) for AITC. Saline $(0.5 \mathrm{ml})$ with $0.1 \%$ Tween 20 and $0.05 \%$ ethanol was used as a vehicle (Veh) for capsaicin. (A and B) Representative traces showing colorectal motility (top) and electromyography (bottom) following intracolonic AITC (1 mM) administration in the presence or absence of A967079 (A96, $0.3 \mathrm{mM}$ ) (A) or intracolonic capsaicin (CAP, $3.3 \mu \mathrm{M}$ ) administration (B). Arrows indicate the time point of drug administration. (C) The bar graphs show AITC-induced $(0.3 \mathrm{mM}, 1 \mathrm{mM}$, and $3 \mathrm{mM}$ ) colorectal contraction or the inhibitory effect of A967079 (0.3 mM) or HCO30031 (0.28 mM) on AITC (at $1 \mathrm{mM}$ ). (D) The bar graphs show cinnamaldehyde-induced (CA, $2 \mathrm{mM})$ colorectal contraction or inhibition by $A 967079(0.3 \mathrm{mM}) .{ }^{*} P<0.05 ;{ }^{* *} P<0.01 ;{ }^{* *} P<$ 0.001 vs. vehicle group; ${ }^{\#} P<0.05$ vs. TRPA1 agonists, $n=4-7$ rats per group, 1 -way ANOVA followed by Bonferroni analysis. (E) The bar graphs show capsaicin-induced ([CAP] $1 \mu \mathrm{M}, 3.3 \mu \mathrm{M}$, and $10 \mu \mathrm{M}$ ) colorectal contraction. ${ }^{*} P<0.05$ vs. vehicle group (Student's $t$ test), $n=5-11$ rats per group. The accumulated AUC value over a 15 -minute period was calculated for colorectal motility analyses. The $\triangle A U C$ value indicates AUC changes after drug administration.

activation in colonic mesenchymal cells results in the induction of PGE2, which might then bind to the EP1 receptor to mediate colorectal contraction.

Abnormal colonic contraction in dextran sodium sulfate-treated animals is associated with increased TRPA1 expression and activation. To elucidate the role of mesenchymal TRPA1 in the pathology of bowel disease, we used a dextran sodium sulfate-induced (DSS-induced) colitis animal model to investigate whether colonic lamina propria TRPA1 is involved in abnormal intestinal motility. After DSS treatment for 7 days, body weight gain was impaired (an increase of $28.2 \%$ for DSS-treated rats and $45.6 \%$ for control rats). In addition, $57 \%$ of DSS-treated rats and $100 \%$ of DSS-treated mice (Trpa1-KO and WT) presented with bloody stool. In contrast to rhythmic colonic contractions observed in water-treated animals, DSS treatment resulted in intense contractions (Figure 6, A and C). These abnormal colonic contractions were inhibited by the TRPA1 antagonists in DSS-treated rats (Figure 6, A and B). The Trpa1-KO mice showed a slight decrease (not statistically significant) or a dramatic reduction (with a significant difference) in colonic motility in normal conditions or DSS-induced colitis conditions, when compared with respective WT mice (Figure 6, C and D).

In support of these data, we found that vimentin-positive cells in the colonic lamina propria were markedly increased in DSS-treated rats compared with that in control rats, suggesting the proliferation of 
A
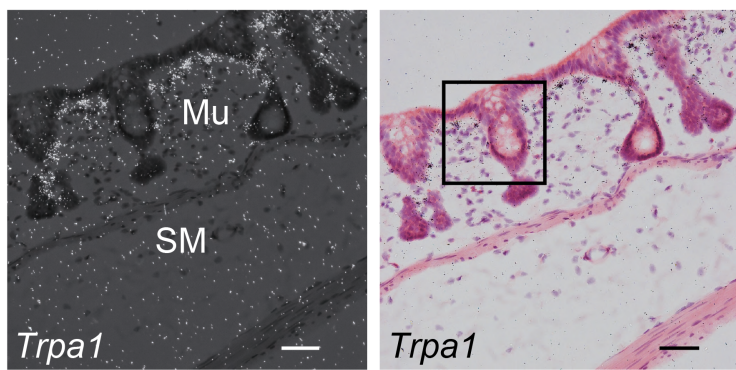

B
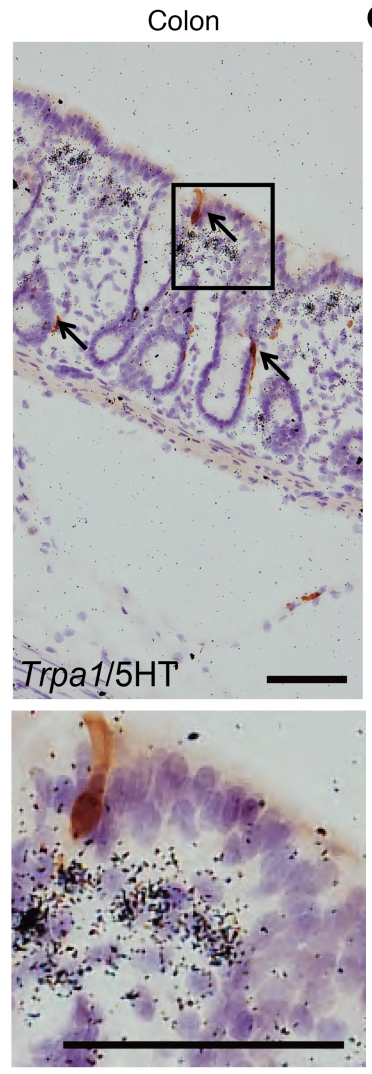

c
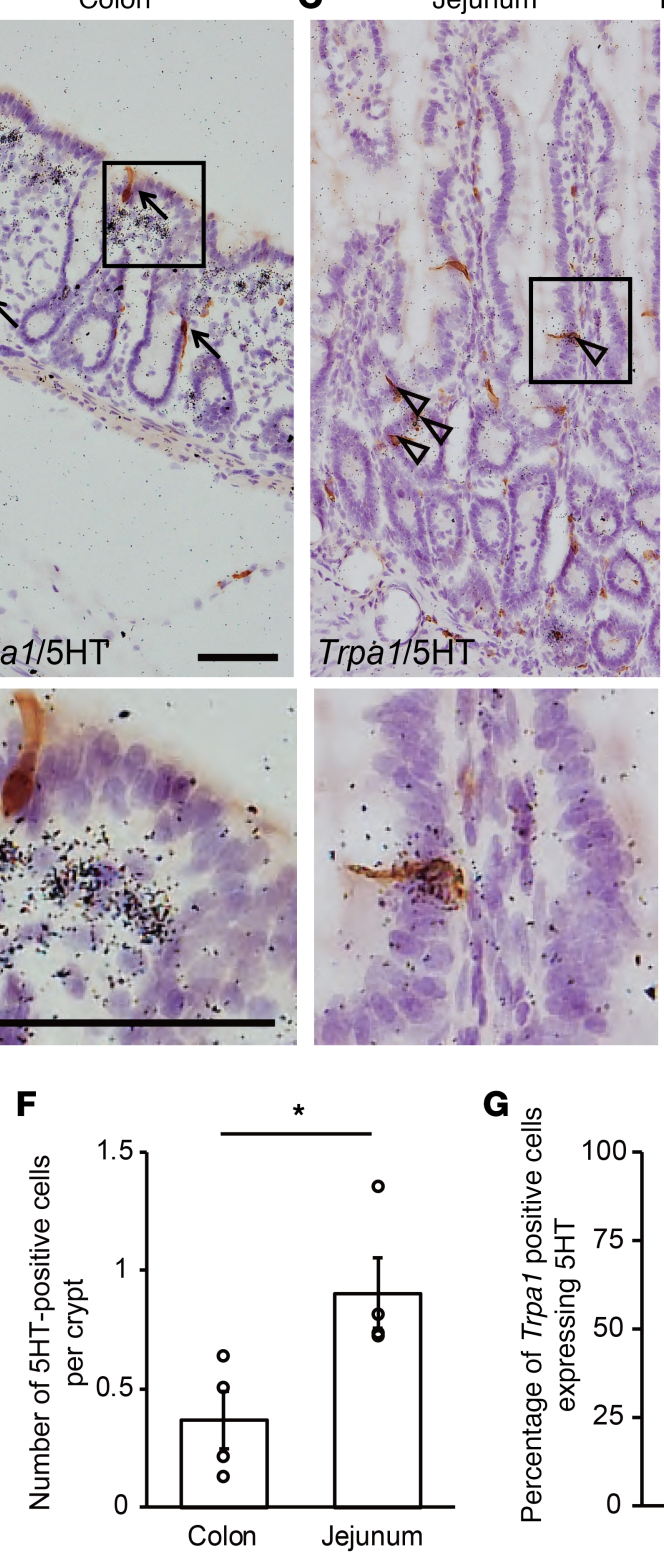
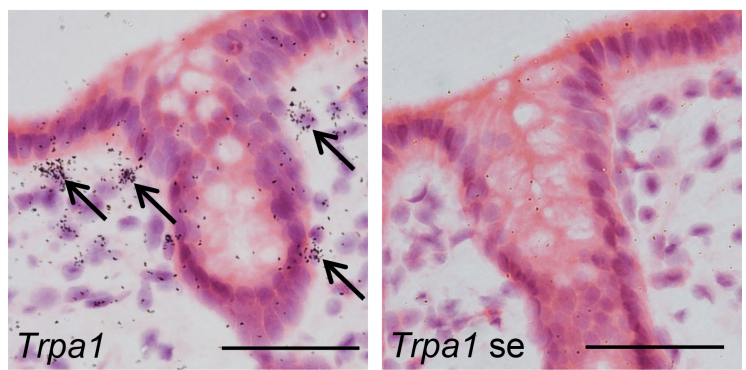

D

Colon

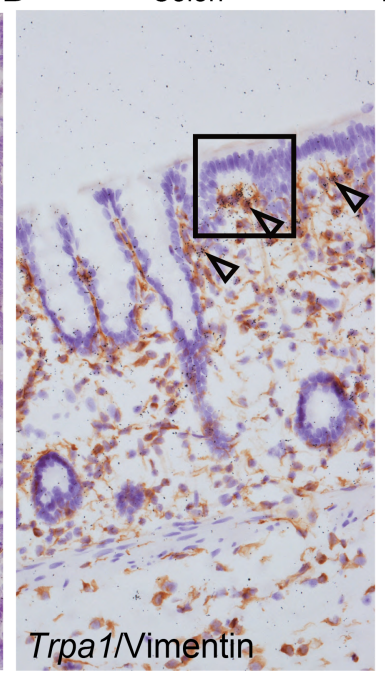

E Jejunum
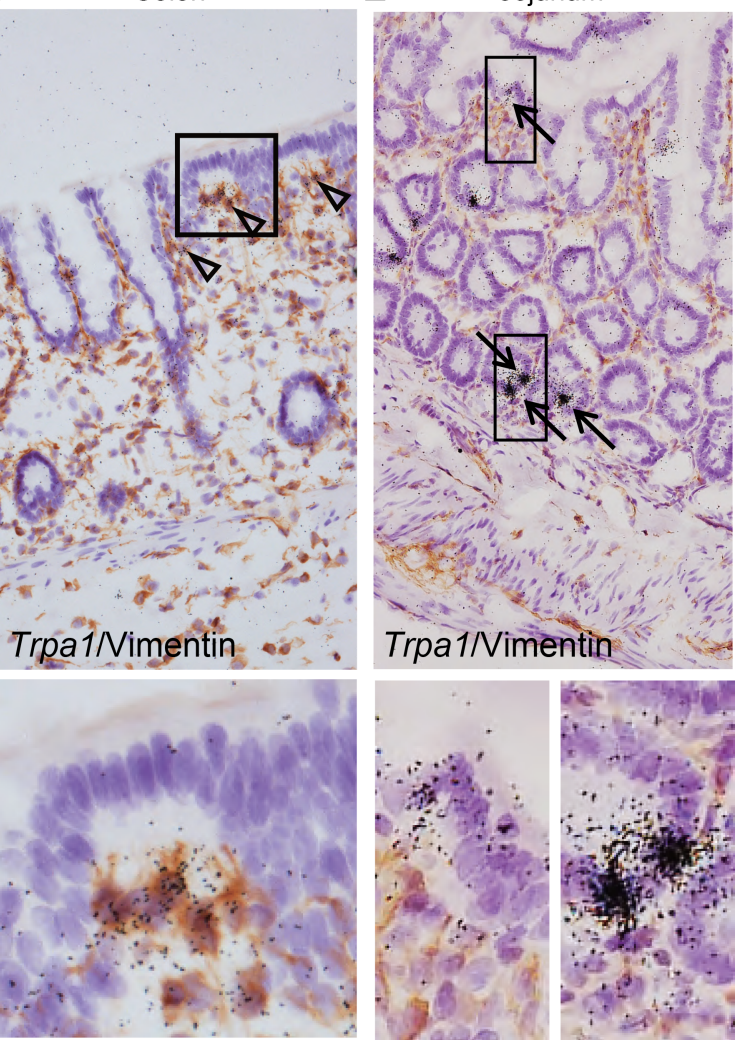
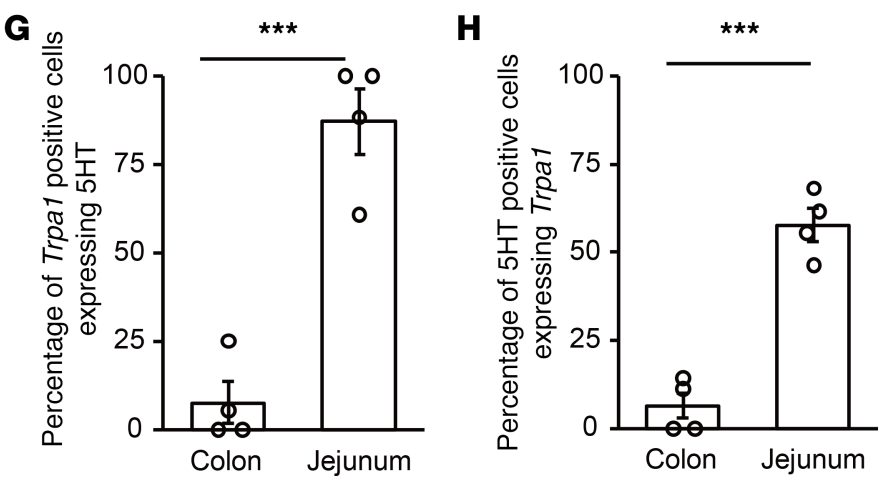

Figure 2. TRPA1 is predominantly expressed by vimentin-positive cells in rat colorectal tissue. (A) Representative in situ hybridization showing Trpa1 mRNA expression in rat colon in dark-field (left, silver grain) and bright-field (middle to right) images. Arrows indicate Trpa1 mRNA signals (silver grain). The right panel shows the control sample using the sense probe. Tissues were counterstained with H\&E. Mu, mucosa; SM, submucosa. (B-E) Representative images of double labeling of Trpa1 mRNA (silver grain, in situ hybridization) and 5-HT or vimentin (brown, immunohistochemistry) in rat colon (B for 5-HT, D for vimentin) and jejunum (C for 5-HT, E for vimentin). Arrows or arrowheads indicating single or coexpression, respectively. Tissues were counterstained with hematoxylin for in situ hybridization. (F) Bar graph showing the number of 5-HT-immunopositive cells per crypt in the epithelial layer of the colon or jejunum tissues. $n=4$ rats/group, ${ }^{*} P<0.05$ vs. colon (Student's $t$ test). (G and $\mathbf{H}$ ) Bar graphs showing percentages of colocalization of $5-\mathrm{HT}$ and Trpa1 (5-HT/Trpa1 in G; Trpa1/5HT in $\mathbf{H}$ ) in the colon or jejunum epithelial tissues from the double labeling. $n=4$ rats/group, ${ }^{* * *} P<0.001$ vs. colon (Student's $t$ test). Scale bars: $50 \mu \mathrm{m}$. 
A

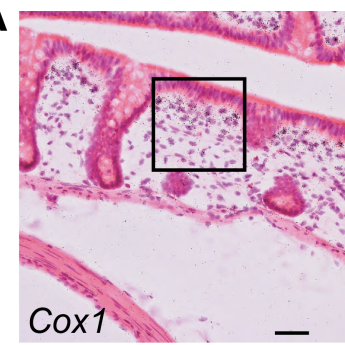

C

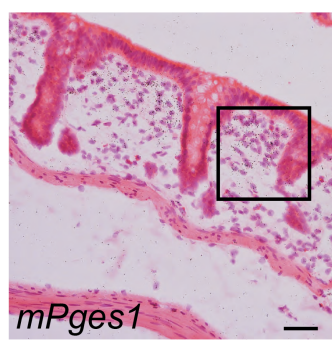

E

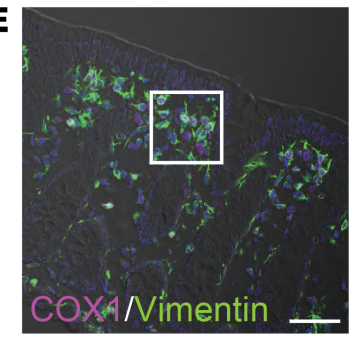

F

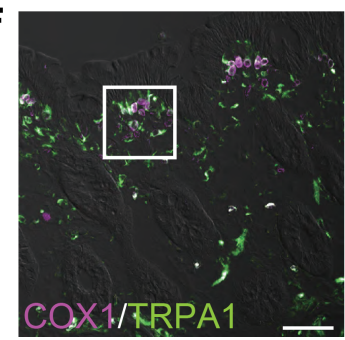

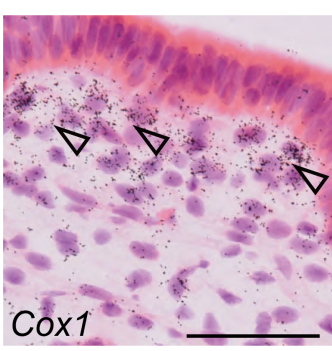
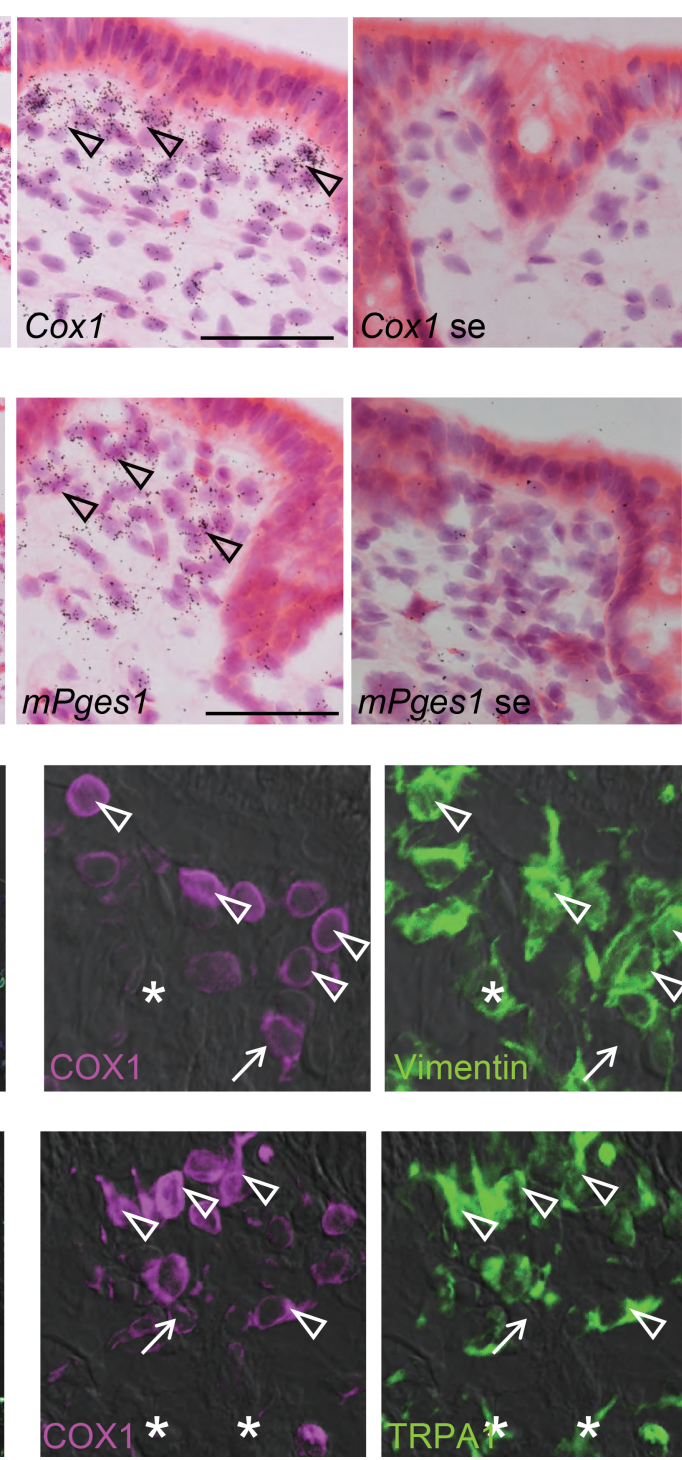

B

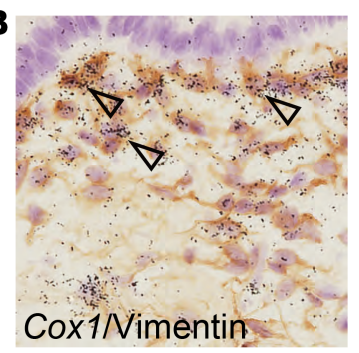

D
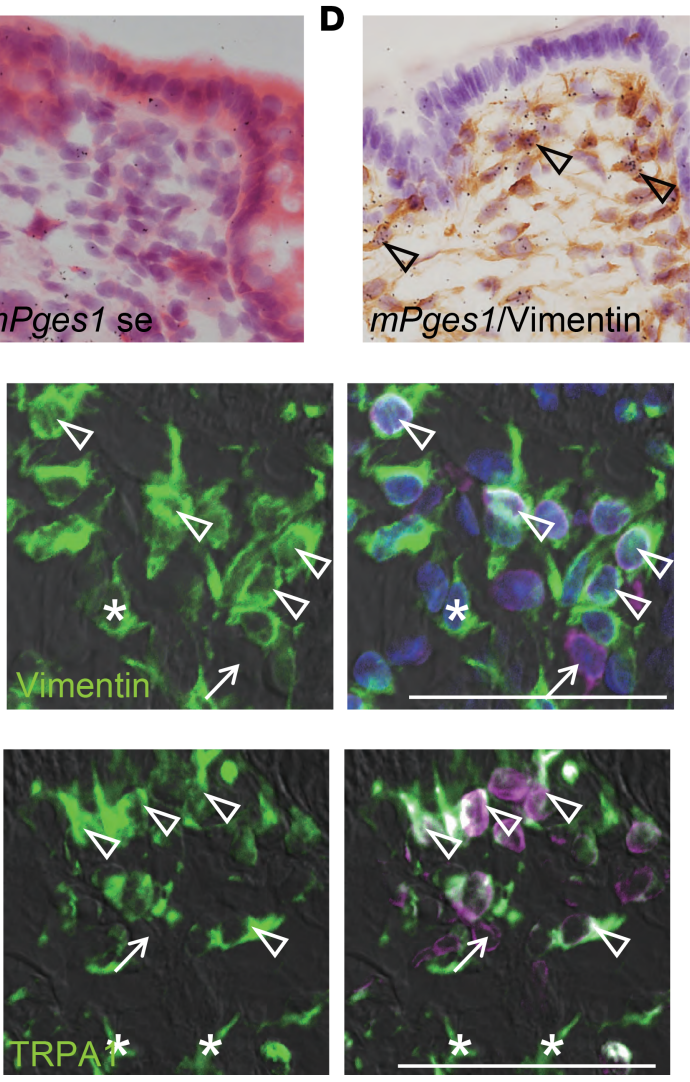

Figure 3. Vimentin-immunoreactive cells express Cox1 and mPges1 in the lamina propria of naive rat colons. (A-D) Representative images showing the labeling of Cox1 or mPges1 mRNA (silver grain) in single ISHH (A or C, respectively) and their double staining with vimentin IHC (brown) (B or D, respectively). Arrowheads depict Cox1 or mPges1 mRNA-positive cells. The right panel in $\mathbf{A}$ or $\mathbf{C}$ shows control samples using their respective sense probe. The tissues were counterstained with H\&E (single ISHH) or hematoxylin (double ISHH-IHC). (E) Double immunofluorescence images of COX1 (magenta) and vimentin (green). Arrowheads show coexpression. Arrows or asterisks represent cells only expressing COX1 or vimentin, respectively. (F) Double immunofluorescence for COX1 (magenta) and TRPA1 (green). Arrowheads indicate coexpression. Arrows or asterisks represent cells only expressing COX1 or TRPA1, respectively. DAPI (blue) was used for nuclear staining. Scale bars: $50 \mu \mathrm{m}$.

mucosal fibroblasts or other cells that recruit mesenchymal cells in the colon during DSS-induced colitis. Trpa1 mRNA-labeled cells were also dramatically increased within these vimentin-positive cells in the colonic lamina propria of DSS-treated rats (Figure 7A). Consistent with histological data, TRPA1 protein levels were found to significantly increase in the colonic mucosa (Figure 7B). Moreover, an oxidative stress mediator, 4-HNE, also known as a TRPA1 endogenous agonist, was upregulated in the colonic lamina propria of DSS-treated mice (Figure 7, C and D). These data suggested that mesenchymal TRPA1 in the mucosa is upregulated and consequently contributes to abnormal colonic motility in DSS-treated animals.

\section{Discussion}

GI motility is a complex process that includes the coordination of gut smooth muscle, autonomous nerves, and enteric nerves, which are associated with several neuronal or hormonal transmitters. In the present study, we identified a subpopulation of mesenchymal cells that expresses TRPA1 in the colonic lamina propria, in 
A
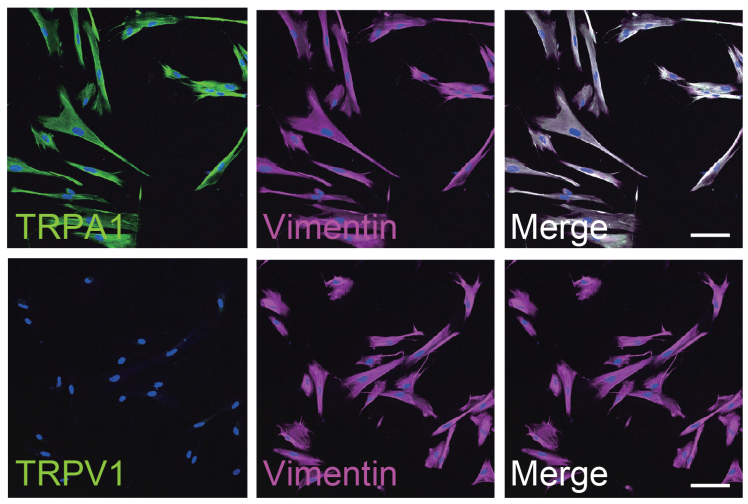

B

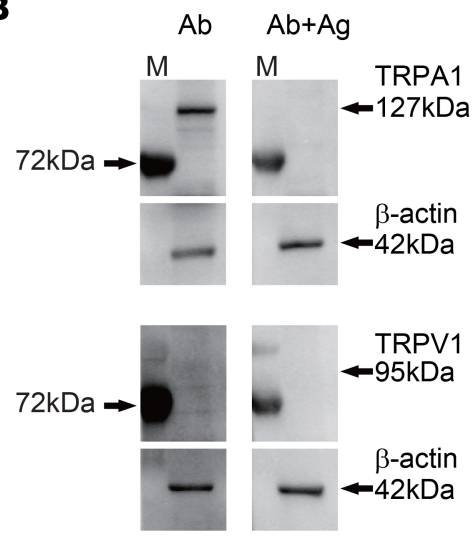

C

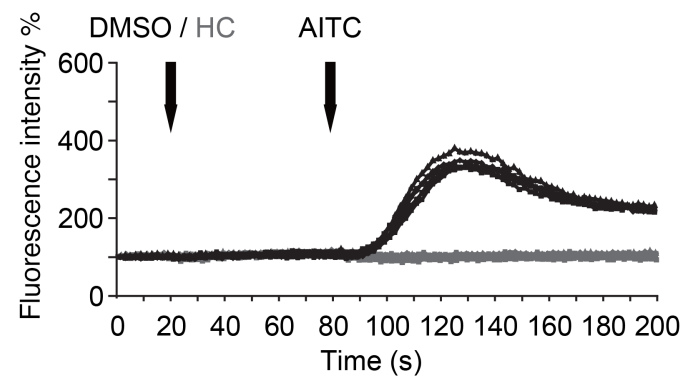

\section{D}

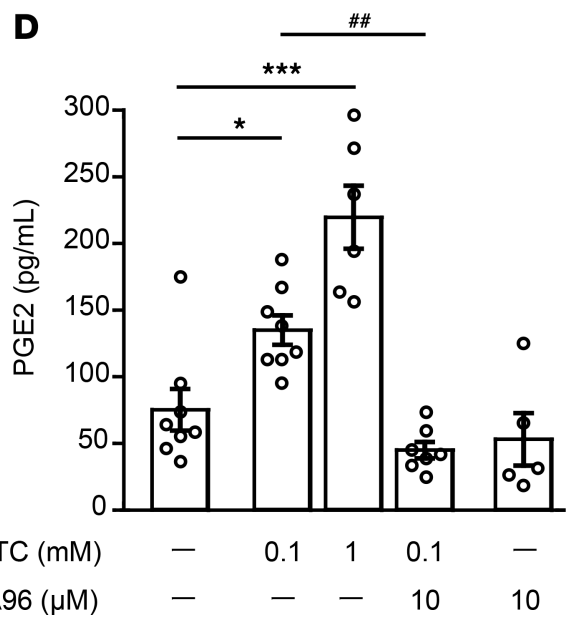

Figure 4. Activation of CCD-18Co cells expressing TRPA1 induces PGE2 release. (A) Double immunofluorescence for TRPA1 (upper image) or TRPV1 (lower image) and vimentin in CCD-18Co cells. Scale bars: $100 \mu \mathrm{m}$. (B) Western blotting showing expression of TRPA1 (top) or TRPV1 (bottom) proteins in CCD-18Co cells. $\beta$-Actin was used for protein normalization. $\mathrm{Ab}$, antibody; $\mathrm{Ab}+\mathrm{Ag}$, antibody preincubated with an antigen; $\mathrm{M}$, a protein weight marker. (C) Calcium imaging data showing the effects of $0.1 \mathrm{mM}$ AITC on intracellular calcium influx in the absence (black lines) or presence (gray lines) of $\mathrm{HC} 030031(\mathrm{HC}, 10 \mu \mathrm{M})$. Arrows show the time point of drug administration $(n=6)$. (D) ELISA data showing the effects of AITC on PCE2 release from CCD-18Co cells. A967079 (A96, $10 \mu \mathrm{M})$ was used as a TRPA1 antagonist. $n=5-8$; ${ }^{*} P<0.05 ;{ }^{* * *} P<0.001$ vs. vehicle group; ${ }^{\#} P<0.01$ vs. AITC (0.1 mM) group (1-way ANOVA followed by Bonferroni analysis).

both rodents and humans. These mesenchymal cells can release PGE2 following TRPA1 activation and consequently regulate colonic motility.

5-HT-expressing EC cells are mainly localized in the epithelial layer and are in contact with the lumen to sense chemical stimuli in the GI tract. The largest stores of 5-HT, harbored by EC cells, are regarded as a major modulator of intestinal motility (19-21). TRPA1, a well-known chemical sensor, has been reported to be expressed in EC cells of the small intestine, and its activation can induce 5-HT release from EC cells to modulate gut motility $(10,11)$. In the present study, we found that 5 -HTexpressing EC cells of the colon, which are distinct from those of the small intestine, do not express TRPA1; this finding suggests that 5-HT does not play a role in AITC-induced colonic contractions. The heterogeneity of EC cells within the GI tract has been noted in recent studies, which showed remarkable regional differences in the expression levels of many genes including those encoding several nutrient receptors and transporters in EC cells from the small intestine and colon (22-24). Enteroendocrine cells show hormonal plasticity in the course of their maturation; many hormones including 5-HT, tachykinin 1, as well as the irritant receptor Trpal gene in EC cells vary their expression depending on the location within the intestine $(12,13)$. The present study showed that both the localization and quantity of 5-HTexpressing cells in the colon differ from those in the small intestine. Specifically, 5-HT-secreting cells were less abundant in the colon than in the small intestine (Figure 2F). In contrast to data suggesting 
$\mathbf{A}$
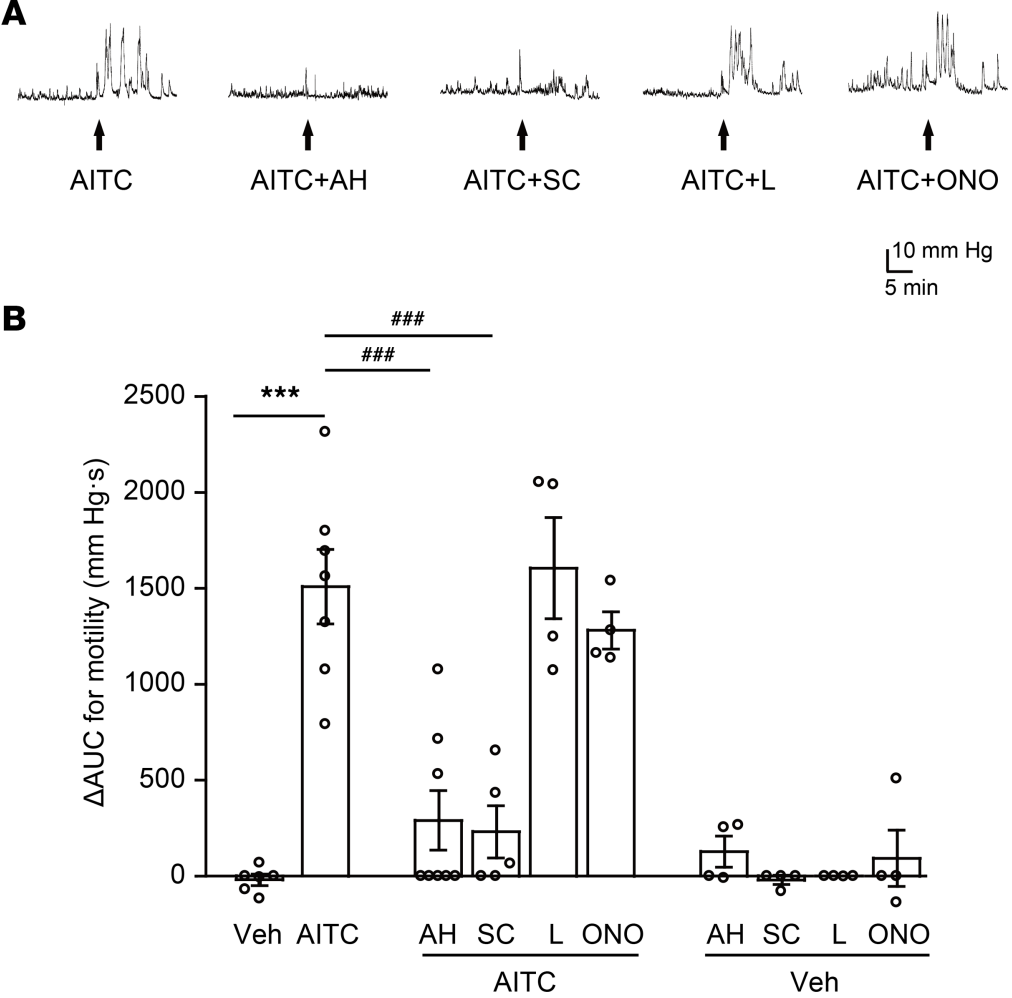

Figure 5. EP1 is involved in intracolonic AITC-induced colorectal contraction in naive rats. (A) Representative traces showing colonic contractile responses following AITC administration in the absence or presence of EP antagonists. Arrows indicate the time point of drug administration. (B) Bar graph showing the effects of EP antagonists on AITC-induced colorectal contraction ( $n=4-8$ rats/group). ${ }^{* *} P<0.001$ vs. vehicle group; ${ }^{* \#} P<0.001$ vs. AITC group (1-way ANOVA followed by Bonferroni analysis). AITC at $1 \mathrm{mM}$ was intracolonically administered. Antagonists of the EP1/2 receptor (AH, $0.15 \mathrm{mg} / 2.5 \mathrm{ml} / \mathrm{kg}$ ), EP1 receptor (SC, $9 \mathrm{mg} / 2.5 \mathrm{ml} / \mathrm{kg}$ ), EP3 receptor (L, $0.54 \mathrm{mg} / 2.5 \mathrm{ml} / \mathrm{kg}$ ), or EP4 receptor (ONO, $0.3 \mathrm{mg} / 2.5 \mathrm{ml} / \mathrm{kg}$ ) were i.p. injected. Saline with $0.8 \%$ DMSO was injected i.p. as the vehicle.

that these cells penetrate the epithelial layer and contact the lumen of the small intestine, most of these cells were found to be distributed in both the crypt and subepithelium in the colon.

In addition to EC cells, we found a subpopulation of lamina propria mesenchymal cells that express TRPA1. These cells also express COX1 and mPGES1, suggesting the ability to produce PGE2. The idea that AITC-induced TRPA1 activation leads to PGE2 release from colonic mesenchymal cells was also supported by our in vitro data. These results showed that PGE2 was released from fibroblasts after AITC stimulation within minutes. In contrast, COX2 might not be involved in AITC-induced colorectal contraction in naive rats, due to the observation of limited COX2 expression in the lamina propria (Supplemental Figure 5).

Among the 4 subtypes of PGE2 receptors in the GI tract, EP1 and EP3 contribute to contraction, whereas EP2 and EP4 contribute to smooth muscle relaxation $(16,25,26)$. In the present study, an EP1 (but not EP3) antagonist completely inhibited AITC-induced colorectal contraction, suggesting that the EP1 receptor is the target of mesenchymal cell-derived PGE2. A previous study demonstrated that EP1 is coexpressed with protein gene product 9.5 (PGP9.5) nerve fibers in the lamina propria of the digestive tract (27). Other reports also indicated that EP1 receptors are highly expressed in the neurons of the myenteric and submucosal ganglia throughout the intestinal tract $(16,17,28)$. These data suggest that EP1 might be expressed by intrinsic afferent neurons of the lamina propria, near TRPA1-expressing mesenchymal cells and mediate PGE2-induced colonic contraction in a neuron-mediated manner. Taken together, following AITC-induced mesenchymal TRPA1 activation, PGE2 might be rapidly released from the mesenchymal cells of the lamina propria and then bind to the neuronal EP1 receptor in the vicinal intrinsic afferents to modulate colonic contraction. In contrast, the TRPA1-EC cell 5-HT theory might be used to explain the modulation of small intestine motility (Figure 8).

A previous study by Poole et al. showed that TRPA1 was expressed in inhibitory motoneurons in the myenteric plexus of mouse intestine and activation of neuronal TRPA1 inhibited the spontaneous 
A

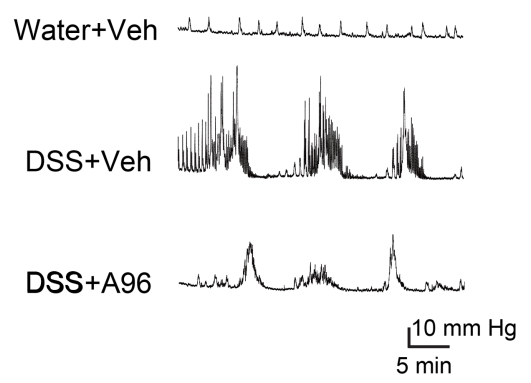

C
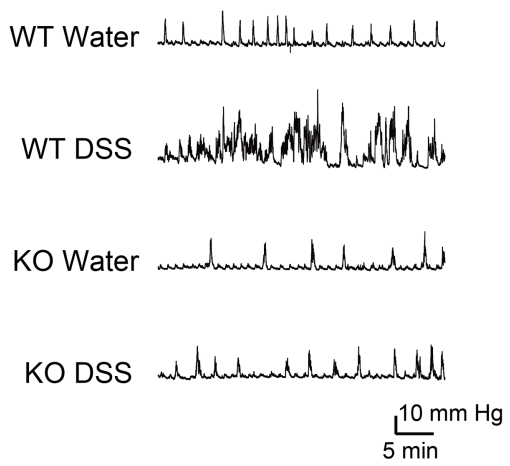

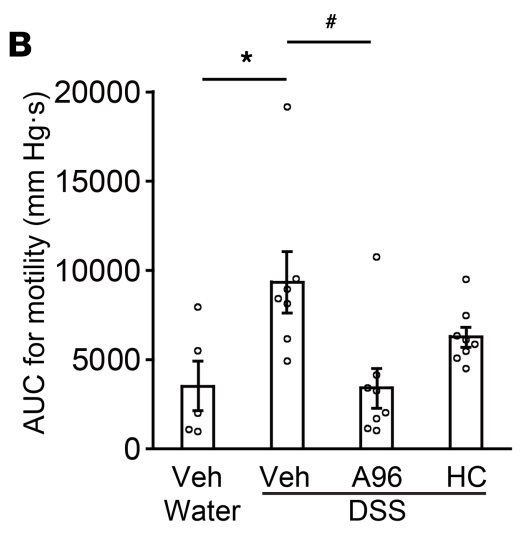

D

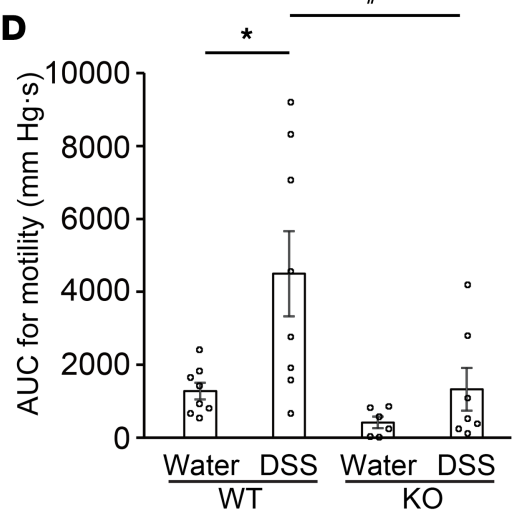

Figure 6. Abnormal colorectal contraction in DSS-treated animals is alleviated by TRPA1 inhibition. (A and B) TRPA1 selective antagonists, $A 967079$ (A96, $40 \mathrm{mg} / 2 \mathrm{ml} / \mathrm{kg}$ ) and HC030031 (HC, $100 \mathrm{mg} / 2 \mathrm{ml} / \mathrm{kg}$ ), were administered by i.p. injection 1 hour before recording. Deionized water with $0.5 \%$ methylcellulose, 10\% DMSO, and 5\% Tween 20 was administered by i.p. injection as the vehicle. (A) Representative traces showing colorectal motilities in rats from the water or DSS-treated groups receiving vehicle (Veh) or A967079 treatment. (B) Bar graph showing the effects of TRPA1 antagonists on colorectal motility in DSS-treated rats ( $n=5-8$ rats/group). ${ }^{*} P<0.05$ vs. water group; ${ }^{\#} P<0.05$ vs. DSS group with vehicle (1-way ANOVA followed by Bonferroni analysis). (C) Representative traces showing colorectal motilities of water- or DSS-treated Trpa1-KO (KO) mice and WT mice. (D) Bar graph showing the effects of TRPA1 on colorectal motility in DSS-treated mice ( $n=6-8$ mice/group). ${ }^{*} P<0.05$, DSS vs. water group in WT mice; ${ }^{\#}<0.05$, Trpa1-KO vs. WT in the DSS-treated group (1-way ANOVA followed by Bonferroni analysis).

neurogenic contraction and transit of the colon (29). We performed double staining of TRPA1 and PGP9.5 and found coexpression indicating TRPA1 was also expressed by myenteric plexus (Supplemental Figure 8); these data are consistent with Poole's study. Poole et al. showed that TRPA1 agonists inhibit colonic contractions in WT mice, an effect that was not observed in Trpa1-KO mice. In addition, intracolonic AITC administration in WT mice prolonged the time required for bead expulsion from the colon, an effect that was not found in Trpa1-KO mice. These data are inconsistent with our findings and the reason could be attributable to the distinct method used. First, the investigators used an isolated tissue (ex vivo) recording system to evaluate intestinal contractility, while we used in vivo models. An in vivo study usually records a real-time response after drug administration and may reflect the actual state of the physiological environment. Second, Poole et al. used AITC at a higher concentration $(0.5 \%$ $=50 \mathrm{mM}$ ) in the colonic transit experiment. High concentrations of AITC could induce an obvious and strong desensitization of TRPA1, which may have the opposite effect on the channels (inhibition) $(30,31)$. Therefore, the delayed colonic bead expulsion in WT mice but not Trpa1-KO mice may be due to the high-concentration-induced desensitization. In contrast, we used AITC at $1 \mathrm{mM}$ in the present study, which is 50 times lower than the concentration used by Poole et al. Therefore, the discrepant findings between the 2 studies may be attributed to the different methodology and/or drug administration.

Intestinal mesenchymal cells of the lamina propria include many cell types such as intestinal fibroblasts, myofibroblasts, pericytes, smooth muscle cells of the muscularis mucosae, and mesenchymal stromal/stem cells $(32,33)$. Considering the morphological features of these mesenchymal cells, these 
A

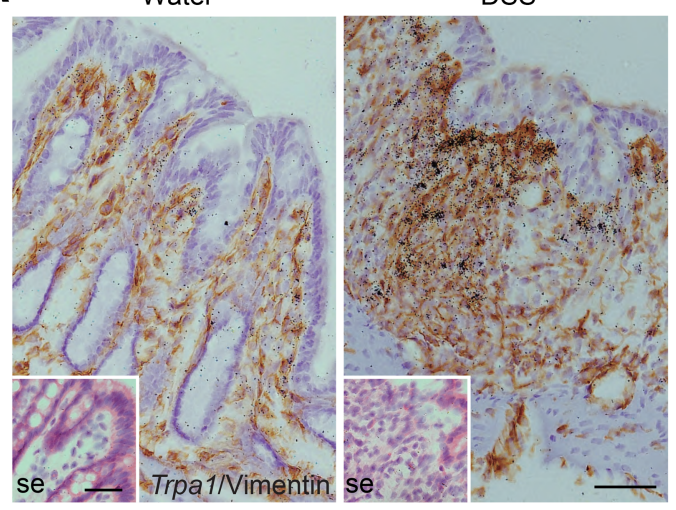

C

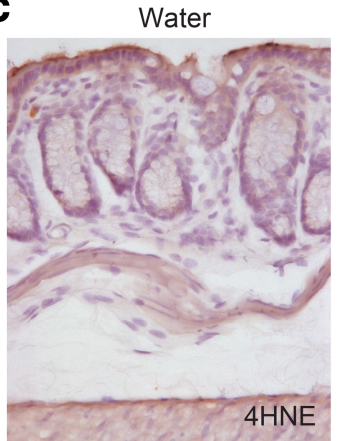

Water

DSS

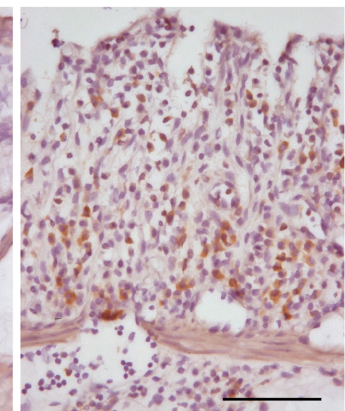

B WaterDSS
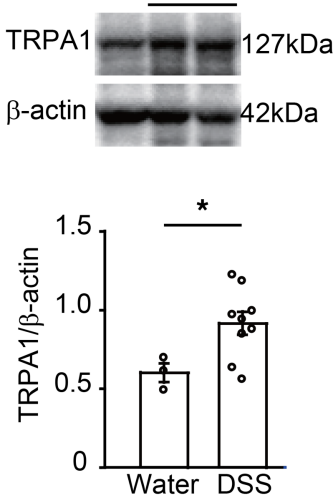

D

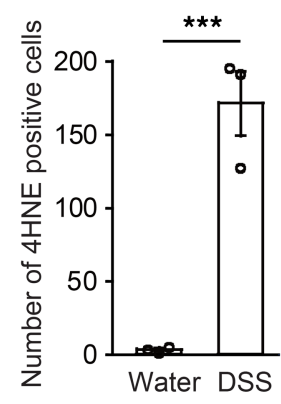

Figure 7. TRPA1 and 4-HNE expression in colonic tissue is increased in DSS-treated animals. (A) Representative images showing double labeling of Trpa1 mRNA (silver grain) and vimentin (brown) in water (left) and DSS-treated rats (right). The tissue was counterstained with hematoxylin. Insets show control images using a sense probe and counterstained with H\&E. Scale bars: $50 \mu \mathrm{m}$. (B) Western blots showing TRPA1 protein levels in colonic tissue of control and DSS rats ( $n=3-9$ rats/group). ${ }^{*} P<0.05$ vs. water group (Student's $t$ test). (C) Representative images showing expression of 4-HNE in water (left)- or DSS (right)-treated mice. Tissues were counterstained with hematoxylin. Scale bar is $50 \mu \mathrm{m}$. (D) Bar graph shows the $4-\mathrm{HNE}$-positive cell numbers in the water and DSS groups. ${ }^{* *} P<0.001$ vs. water group (Student's $t$ test)

TRPA1-expressing cells are most likely fibroblasts. The role of these cells in physiology and disease remain poorly understood (34). Our data thus provide evidence that these cells not only act as supporting cells but also play an important role in regulating intestinal functions. A previous study by Kurahara et al. showed that intestinal myofibroblasts in patients with Crohn's disease express TRPA1 and a myofibroblast cell line responds to AITC (35). However, they did not characterize TRPA1expressing cells using the myofibroblast marker $\alpha$-SMA in either naive animals or in healthy humans. In our present study, we did not detect any coexpression of TRPA1 with $\alpha$-SMA in naive rats (see Supplemental Figure 2D). These data do not deny that TRPA1-expressing fibroblasts may be activated during colitis and differentiate into myofibroblasts with upregulated TRPA1. In most organ systems, cell signals initiated after tissue-specific insult lead to differentiation of fibroblasts to the myofibroblast phenotype (36). Therefore, in patients with IBD, the TRPA1-expressing fibroblasts may be activated and transform to myofibroblasts.

Due to the different functions of various parts of the GI tract, the modes of motility can vary. In the small intestine, motility is modulated by the state of digestion. Digesta enters the small intestine and stimulates hormones, enteric neurons, and extrinsic neurons to coordinate motility for nutrient absorption. Thus, motility of the small intestine is stimulated by chemicals and is associated with a slow and regulated propulsion rate. In the colon, water and electrolyte absorption turns semifluid digesta into solids. Thus, colon motility requires extensive turnover and a slow transit rate, whereas defecation requires rapid propulsion over a brief period of time (37). Therefore, the contraction process is predominant in the colon. The distinct expression of TRPA1 as well as its coexpression with 5-HT or PGE2 in the small and large intestine might reflect different modalities of bowel movement. 


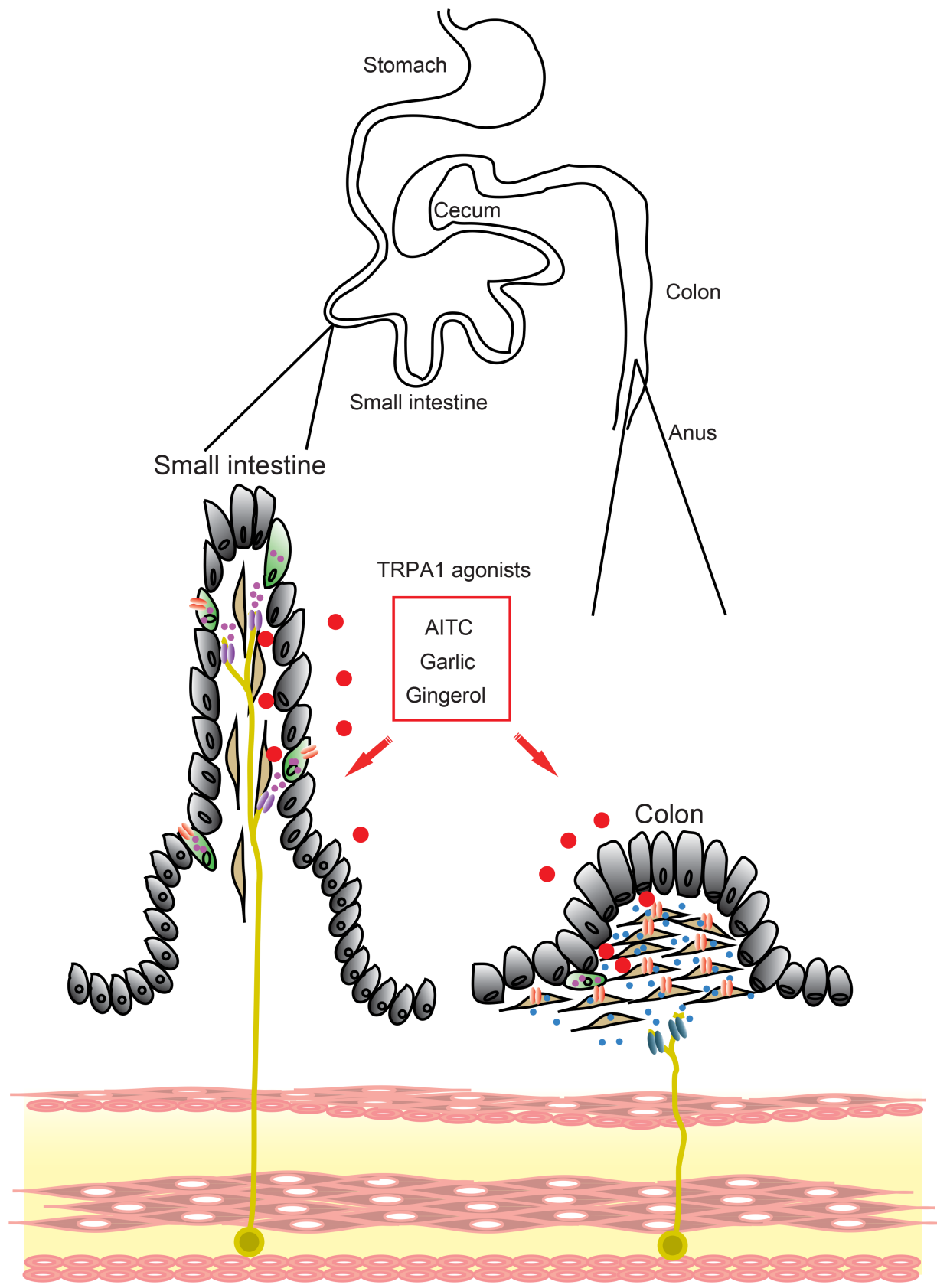

Epithelial layer

Lamina propria

Muscularis mucosa

Submucosa

Circular muscle

Myenteric plexus

Longitudinal muscle

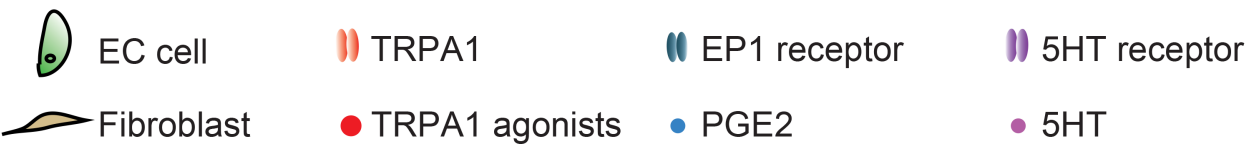

Figure 8. The potential involvement of TRPA1 in intestinal motility in the small and large intestine. The expression of TRPA1 is different in the small intestine and colon. In the small intestine, TRPA1 is expressed in EC cells located in the epithelium and crypts. TRPA1 activation by chemicals in foods induces 5 -HT release from EC cells to regulate intestinal motility. In the colon, TRPA1 is predominantly expressed in fibroblast-like mesenchymal cells that are localized to the lamina propria. Exogenous agonists from the lumen or endogenous agonists from mucosal tissues might activate TRPA1 and induce PGE2 release from these cells and consequently modulate colorectal motility. These events might involve intrinsic afferent-expressing 5-HT receptors in the small intestine and the PGE2 receptor EP1 in the large intestine, respectively.

IBD comprises typical disorders of the large intestinal tract. In patients with IBD, inflammation mainly damages the colon and rectum, although it involves the entire bowel. For example, patients with ulcerative colitis present with diffuse damage to the mucosa from the rectum to the colon. The DSS animal model used in this study presents with signs such as diarrhea, abdominal pain, hematochezia, and weight loss, 
which closely resemble the clinical features of human ulcerative colitis (38-40). Consistent with previous studies indicating that inflammation can induce vimentin-positive fibroblast proliferation in the mucosa of the GI tract $(41,42)$, we confirmed a remarkable increase in vimentin-positive cells as well as with TRPA1 coexpression in the colonic mucosa of DSS rats (Figure 7, A and B). Upregulation of endogenous TRPA1 agonists such as 4-HNE (confirmed in this study; Figure 7, C and D) or $\mathrm{H}_{2} \mathrm{O}_{2}$ was observed in DSS-treated rats in previous studies $(43,44)$. These molecules might stimulate mesenchymal TRPA1 and induce extensive PGE2 release, consequently enhancing colonic contraction, causing diarrhea and/or abdominal pain in these animals. The data found in this study, indicating that pharmacological or genetic blockage of TRPA1 significantly reduces abnormal GI motility in DSS-induced animals, suggest a vital role for TRPA1 in the pathologic colonic motility associated with IBD.

Our results focused on the distinct localization of TRPA1 in the small intestine and colon and found that this protein is expressed by EC cells and mesenchymal cells, respectively. TRPA1 acts as a modulator for GI motility in both the small and large intestine. In addition to the previously reported role of the EC cell 5-HT axis in the small intestine, we demonstrated that TRPA1-mediated PGE2 release from mesenchymal cells near the epithelium might represent a novel mechanism of colonic contraction. These observations will provide the basis for the development of new drugs that target TRPA1 or EP1 for colonic motility-related symptoms such as diarrhea and constipation.

\section{Methods}

Animals. Adult male Sprague-Dawley rats (6 to 8 weeks old) or C57BL/6 mice (7 to 12 weeks old) (Japan SLC Inc.) were used for the experiments. Trpa1-KO mice were provided by Makoto Tominaga (Okazaki Institute for Integrative Bioscience, NINS, Okazaki, Japan) from David Julius (UCSF, San Francisco, California, USA). (4) In experiments comparing WT and Trpa1-KO mice, the WT mice were obtained from the offspring of purchased female C57BL/6 mice, which have the same genetic background as Trpa1KO mice. The Trpal-KO mice were obtained by inbreeding the parental mice. All mice were bled at the same specific pathogen-free area and received the same feedings in the same environment to control the microbiota. Rats or mice were housed in plastic cages and were provided with water and food ad libitum at room temperature $\left(23^{\circ} \mathrm{C} \pm 1^{\circ} \mathrm{C}\right)$ with a 12 -hour light-dark cycle (lights on at $7 \mathrm{am}$ ). For naive rat and mouse recordings, animals in each group were fasted for 24 hours before recording colorectal motility. For DSS-model rats, 4\% DSS (36-50 kDa, MP Biomedical) was prepared with sterilized tap water to replace drinking water and administered to rats for 7 consecutive days. DSS-model rats were used on day 8. Control rats received sterilized tap water. Due to the severe damage and high mortality rates of $4 \%$ DSS-treated mice, we used 1.5\% DSS for the mouse experiments. The mice were administered DSS for 7 days and were used on day 8.

Colorectal motility and visceral pain response recording. Rat colorectal motility was measured using a pressure sensor that was connected to an inserted balloon, as shown in Supplemental Figure 1A. Specifically, after the inhalation of $2 \%$ isoflurane (Wako Pure Chemical Corporation), i.v. drug delivery was established in animals. Rats were anesthetized via an i.v. infusion of $0.5 \%$ propofol (Maruishi Pharmaceutical) at 5 $\mathrm{ml} / \mathrm{h}$ for 5 minutes, which was maintained at a dose of $7.5 \mathrm{ml} / \mathrm{h} / \mathrm{kg}$. For mice, $0.5 \%$ propofol at 124.8 $\mathrm{ml} / \mathrm{h} / \mathrm{kg}$ was used for 2.5-5.5 minutes to establish deep sedation and maintained at a dose of $22 \pm 1$ $\mathrm{ml} / \mathrm{h} / \mathrm{kg}$. A $4-\mathrm{cm}$ (for rats) or 1-cm (for mice) slack latex balloon (Okamoto, Tokyo, Japan) was inserted into the intestine $5 \mathrm{~cm}$ (rats) or $2 \mathrm{~cm}$ (mice) from the anus to detect colorectal motility. Warm $\left(37^{\circ} \mathrm{C}\right)$ water was infused into the balloon to fill the enteric cavity and obtain a pressure of $10 \pm 2 \mathrm{mmHg}$ (rats) or $3 \pm 2$ $\mathrm{mmHg}$ (mice) to observe colorectal motility (Supplemental Figure 1B). The colorectal movement rhythm was sensed by a BP transducer (AD Instruments) and recorded using a PowerLab 8/35 with a Bio Amp (AD Instruments). Meanwhile, the visceral pain response after drug administration was assessed by recording electromyography of the visceromotor response (VMR), as reported previously (45). After a 30- to 60-minute adaptation period, the recording was used for analyses. For naive rats, the TRPA1 agonist-induced colorectal motility was analyzed based on the AUC $(\mathrm{mmHg} \cdot \mathrm{s})$ of the intracolonic pressure signal above $20 \mathrm{mmHg}$, and drugs were intracolonically administered or delivered via i.p. injection during the recording. During intracolonic administration, an animal feeding needle was inserted into the intestine 4 to $5 \mathrm{~cm}$ from the anus and the drugs or vehicles $(0.5 \mathrm{ml})$ were gently intracolonically administered. The difference in integrated AUC values (for $15 \mathrm{~min}$ ) between pre- and postdrug administration was used to evaluate the effects of drugs on naive rats (Supplemental Figure 1B). For DSS-treated animals, the spontaneous 
colorectal motility was analyzed based on the AUC of the intracolonic pressure signal above $10 \mathrm{mmHg}$ (for rats) or $5 \mathrm{mmHg}$ (for mice). The TRPA1 antagonists were delivered via i.p. injection 1 hour in rats before recording. The integrated values of AUC for 30 minutes were used to analyze DSS-treated animals. VMR to colorectal distention (CRD) was performed in mice as a positive control of electromyography recordings. CRD was performed by tonic inflation up to $60 \mathrm{mmHg}$ over a period of 10 seconds. VMR to CRD was quantified as the value of the AUC $(\mu \mathrm{V} \cdot \mathrm{s})$ of electromyography after distention for 10 seconds. The AUC immediately before distention for 10 seconds was used as baseline. Differences in AUC values from the baseline were used for data analysis.

Animal tissue and frozen section preparation. After undergoing deep anesthesia, the rats or mice were perfused transcardially with PBS (for mice) or $1 \%$ paraformaldehyde (Nacalai Tesque for rats) followed by $4 \%$ paraformaldehyde. Rat colorectal tissue $(4-10 \mathrm{~cm}$, from the anus) and the jejunum were then removed. Using the Swiss-roll technique, mouse colorectal tissue from the anus to the cecocolic junction was prepared. The sections were post-fixed in $4 \%$ paraformaldehyde at $4^{\circ} \mathrm{C}$ for 24 hours and then transferred to $30 \%$ sucrose at $4^{\circ} \mathrm{C}$ for 3 days. Samples were finally embedded in Tissue-Tek O.C.T. (Sakura Finetek) and frozen. Frozen sections (10 to $12 \mu \mathrm{m}$ ) were used for immunostaining and in situ hybridization.

Human tissue preparation. We obtained endoscopic biopsy samples from the rectum of 3 healthy subjects (aged 61-84 years) using biopsy forceps (Radial Jaw 4, Boston Scientific Corporation). Immediately after biopsy, the samples were post-fixed in $4 \%$ paraformaldehyde at $4^{\circ} \mathrm{C}$ for 3 hours, which was then replaced with $30 \%$ sucrose in PBS for 2 days at $4^{\circ} \mathrm{C}$. Rectum sections $(12 \mu \mathrm{m})$ were cut using a cryostat and processed for immunohistochemistry.

In situ hybridization histochemistry and double labeling combined immunohistochemistry with in situ hybridization histochemistry. The expression and distribution of mRNA encoding Trpa1, Cox1, Cox2, and mPges 1 in the colorectal mucosa were detected using single in situ hybridization histochemistry (ISHH) or that combined with immunohistochemistry (IHC) for vimentin. For ISHH, clones (pCRII-TOPO, Invitrogen) containing a partial sequence corresponding to the coding regions of Trpa1, Cox, and mPges1 were prepared (Supplemental Table 1). Using the enzyme-digested clones, ${ }^{35}$ SUTP-labeled antisense and sense cRNA probes were synthesized. The ${ }^{35} \mathrm{~S}$-labeled probes in the hybridization buffer were placed on the section and then incubated at $55^{\circ} \mathrm{C}$ overnight ( $>18$ hours). After the hybridization reaction, slides were coated with NTB emulsion (Kodak) and exposed for 2-8 weeks. Once developed in D-19, the sections were stained with H\&E and placed on coverslips.

To examine the distribution of Trpa1, Cox1, Cox2, and mPges 1 mRNA, we used a double-labeling method combining IHC with ISHH as reported previously.(46) The following antibodies were used: mouse anti-vimentin antibody (1:2000, Millipore) and goat anti-5-HT antibody (1:1000, Immunostar Inc.). After the hybridization reaction, the slides were coated with NTB emulsion and exposed for 3-10 weeks. Once developed in D-19, the sections were stained with H\&E and coverslipped. Images were acquired using a microscope (Nikon Eclipse 80i, Nikon Instruments) with a Nikon Ds-Ri1 camera using a plan Apo $\times 20$ or $\times 40$ objective lens.

For double ISHH image analysis, 3 areas $\left(207.57 \times 166.05 \mu \mathrm{m}^{2}\right)$ were randomly acquired from each animal's intestine sample $(n=4)$ at $\times 40$ magnification. At the same magnification and exposure level, the background areas $\left(207.57 \times 166.05 \mu \mathrm{m}^{2}\right)$ were acquired from each slide. Using NIH ImageJ 1.52a (Wayne Rasband, National Institutes of Health; http://rsb.info.nih.gov/ij/), the upper and lower thresholds of gray-level density were set such that only silver grains were accurately discriminated in the background image, and the number of dots per $\mu \mathrm{m}^{2}$ was calculated as a background level. We defined a 30 -fold increase of background level on the cell nucleus as the borderline between positive and negative cells for the Trpa1 probe. The total number of Trpa1-positive cells in rat colon and jejunum mucosa (excepted muscularis mucosae) were then counted. The percentage of Trpa1-positive cells that coexpressed with vimentin or 5-HT was used for data analysis.

Tissue immunofluorescent staining. The expression of TRPA1, vimentin, $\alpha-S M A, C D 45$, CD31, 5-HT, COX1, COX2, SP, CGRP, and PGP9.5 in the rat colonic mucosa was examined by double-immunofluorescent staining. Frozen tissue sections were blocked using 10\% normal animal serum for 1 hour and then incubated with a mixture of primary antibodies (Supplemental Table 2) for 2 days at $4^{\circ} \mathrm{C}$. This step was followed by incubation with a mixture of Alexa 488-conjugated and 594-conjugated secondary antibodies (Supplemental Table 3) overnight at $4^{\circ} \mathrm{C}$. For TRPA1 and COX1 double staining, anti-TRPA1 antibody diluted at 1:250,000 was used according to tyramide signal amplification (TSA kit NEL701A001, PerkinElmer) fluorescence procedures and then combined with a COX1 antibody at 1:5000. A detailed TRPA1 
and PGP9.5 double-staining method is described in the legend of Supplemental Figure 8. In our experiments, no positive signal was observed using this concentration without TSA amplification. The sections were mounted with DAPI (Vector Laboratories). For our immunofluorescence staining experiments, all negative controls (with only primary or secondary antibodies; TRPA1 antibody absorption with antigen) were confirmed. Images were acquired using a confocal laser-scanning microscope (FV10-ASW Version 03.01.02.02; Olympus Corporation) with a water Plan-Neofluar $\times 20$ or $\times 40$ objective lens.

Single immunohistochemistry. Antigen retrieval was performed using Histo TV (Nacalai Tesque) at $70^{\circ} \mathrm{C}$ for 30 minutes. Endogenous peroxidase was blocked with $0.3 \% \mathrm{H}_{2} \mathrm{O}_{2}$ at room temperature for 30 minutes. After blocking with $10 \%$ normal goat serum at room temperature for 1 hour, the tissue section was incubated with a rabbit anti-4-HNE (Supplemental Table 2) for 1 day at $4^{\circ} \mathrm{C}$. A biotinylated anti-rabbit $\operatorname{IgG}(\mathrm{H}+\mathrm{L} ; 1: 400$, Vector laboratories, BA-1000) was used as a secondary antibody overnight at $4^{\circ} \mathrm{C}$, and target antigen signal was detected using an avidin-biotin complex (strep ABC peroxidase kit, Nacalai Tesque). The section was visualized with DAB (Wako). Images were acquired using a microscope (Nikon eclipse 80i, Nikon Instruments) with a Nikon Ds-Ri1 camera using a Plan Apo $\times 40$ objective lens. Mouse colorectal tissue $(1-5 \mathrm{~cm}$ from anus) was used to acquire images. For mice in the DSS group, 3 imaging areas from epithelial cells and damaged crypt areas were used to count 4-HNE-positive cells. For water-treated mice, 3 random areas were used for cell counting because no damage was observed. The total 4-HNE-positive cell numbers were used for data analysis.

Human colonic fibroblast culture. CCD-18Co cells were cultured in Eagle's minimal essential medium with $10 \%$ FBS, $1 \times$ penicillin, and streptomycin.

Cell immunostaining. CCD-18Co cells were subcultured on coverslips for 2 days and then fixed with 4\% paraformaldehyde for 30 minutes. After blocking with 10\% normal goat serum for 1 hour, samples were incubated with rabbit anti-TRPA1 (1:100, Alomone Labs), rabbit anti-TRPV1 (1:100, Alomone Labs), and mouse anti-vimentin (1:1000, Millipore) for 24 hours at $4^{\circ} \mathrm{C}$. The immunofluorescence signals were obtained using Alexa Fluor 594- or Alexa Fluor 488-conjugated secondary antibodies at 1:1000 (Molecular Probes) for 1 hour at $4^{\circ} \mathrm{C}$. Images were acquired using a confocal laser scanning microscope (FV10ASW Version 03.01.02.02, Olympus Corporation) with the water Plan-Neofular $\times 20$ objective lens.

Calcium imaging. Fluo-4 acetoxymethyl ester (DoJindo Molecular Technologies) at $2 \mu \mathrm{M}$, prepared in Hank's buffered salt solution containing $2 \mathrm{mM}$ probenecid (Sigma-Aldrich), was used with CCD-18Co cells for 1 hour at $37^{\circ} \mathrm{C}$. Intracellular calcium was then measured at an excitation wavelength of $485 \mathrm{~nm}$ and an emission wavelength of $525 \mathrm{~nm}$ using a Flex Station III (Molecular Devices).

PGE2 assay. CCD-18Co cells were seeded in 24-well plastic culture plates at a density of $8 \times 10^{4}$ cells/ $\mathrm{ml}$ and incubated at $37^{\circ} \mathrm{C}$ with $5 \% \mathrm{CO}_{2}$ for 24 hours. Culture medium was then replaced with Eagle's minimal essential medium supplemented with $1 \%$ FBS for 48 hours before administering stimulants to cultures. Cells were incubated with $0,0.1$, or $1 \mathrm{mM}$ AITC at room temperature for 3 minutes. A967079 $(10 \mu \mathrm{M})$ was administered for 1 minute prior to AITC $(0.1 \mathrm{mM})$ stimulation. Supernatants were harvested for ELISA assay using the Prostaglandin E2 EIA (Cayman Chemical) according to the manufacturer's protocol, and $0.01 \%$ DMSO was used as the vehicle.

Western blotting. Western blotting was performed as described in a previous study (47). For CCD-18Co cells, total protein from the cells was extracted in $500 \mu \mathrm{l}$ of cold lysis buffer $(1 \% \mathrm{NP} 40,20 \mathrm{mM}$ Tris- $\mathrm{HCl}$, $150 \mathrm{mM} \mathrm{NaCl}, 1 \mathrm{mM}$ EDTA, and 10\% glycerol) with proteinase and phosphatase inhibitors. For colonic tissue, the mucosa layer was dissociated from the rat colon, and the total protein from the mucosal tissue was collected. These protein samples were separated on 10\% SDS-PAGE gels and then transferred to PVDF membranes (Immobilon-P, Millipore) using a mini trans-blot cell (Bio-Rad Laboratories). The membranes were probed with rabbit anti-TRPA1 (1:500, Alomone Labs), rabbit anti-TRPV1 (1:500, Alomone Labs), and mouse anti- $\beta$-actin (1:1000, Sigma-Aldrich) antibodies. The control antigen was preincubated with antibody at a ratio of $1 \mu \mathrm{g}$ of peptide per $1 \mu \mathrm{g}$ of antibody for 30 minutes at $4^{\circ} \mathrm{C}$ with rotation. An alkaline phosphatase-conjugated AffiniPure $\mathrm{F}\left(\mathrm{ab}^{\prime}\right) 2$ fragment donkey anti-mouse IgG $(\mathrm{H}+\mathrm{L})$ (1:5000, Jackson ImmunoResearch Laboratories) secondary antibody and an alkaline phosphatase-conjugated mouse anti-rabbit IgG (light chain-specific; 1:5000, Jackson ImmunoResearch Laboratories) secondary antibody were used to detect immunoreactive bands. The bands were visualized with CDP-Star (Roche Applied Science).

Reagents. The TRPA1 agonist, AITC, was obtained from Nacalai Tesque. Cinnamaldehyde was obtained from Sigma-Adrich. Two TRPA1 antagonists, HC-030031 and A967079, were both from Alomone labs. The TRPV1 agonist capsaicin was from Sigma-Aldrich. PGE2 was from Nacalai Tesque. An EP1/2 receptor antagonist, AH6809, was from Abcam. The selective EP1 receptor antagonist SC19220 
was from Sigma-Aldrich. The EP3 receptor antagonist L-798106 was from Tocris Bioscience. An EP4 receptor antagonist, ONO-AE3-208, was from Cayman Chemical.

Statistics. All results are expressed as mean \pm SEM. Two-sided tests were used. A Student's $t$ test was applied to compare 2 groups. One-way ANOVA with Bonferroni analysis was applied for multigroup analyses. Differences were accepted as significant when $P<0.05$. Sigmoidal curve fitting to determine $\mathrm{EC}_{50}$ was performed using Origin 9 (Originlab).

Study approval. All animal experimental procedures were approved by the Hyogo University of Health Sciences Committee on Animal Research (Kobe, Japan; approval no. 2016-08; 2017-24) and were performed in accordance with the NIH guidelines on animal care. The experiment using human colorectal tissue staining was performed in accordance with human ethics regulations (Hyogo College of Medicine: Nishinomiya, Japan, approval no. 0281), and written informed consent was obtained.

\section{Author contributions}

YY, SW, and KK performed the main experiments and carried out the analyses. YH performed the preliminary experiments. HK performed IHC and ISHH staining. TK performed human experiments. HY and YK took part in the experimental design. SW and YD designed the experiments and drafted the manuscript. SY, JL, HM, and KN contributed to critical revision of the manuscript for important intellectual content. $\mathrm{KN}$ and YD supervised the project.

\section{Acknowledgments}

This work was supported by Grants-in-Aid for Scientific Research KAKENHI 25893280 and 17K09048, the Ministry of Education, Culture, Sports, Science and Technology Research Basis Formation Supporting Project for Private University S1411041, the CMCIHCM Research Grants, and the HCM-HUHS collaboration research grants.

Address correspondence to: Yi Dai, Department of Pharmacy, School of Pharmacy, Hyogo University of Health Sciences, 1-3-6 Minatojima, Chuo-ku, Kobe, Hyogo 650-8530, Japan. Phone: 81.78.304.3147; Email: ydai@huhs.ac.jp. Or to: Koichi Noguchi, Department of Anatomy and Neuroscience, Hyogo College of Medicine, Nishinomiya, Hyogo 663-8501, Japan. Phone: 81.798.45.6415; Email: noguchi@hyo-med.ac.jp.

1. Hansen MB. Neurohumoral control of gastrointestinal motility. Physiol Res. 2003;52(1):1-30.

2. Dai Y. TRPs and pain. Semin Immunopathol. 2016;38(3):277-291.

3. Zhang XF, Chen J, Faltynek CR, Moreland RB, Neelands TR. Transient receptor potential A1 mediates an osmotically activated ion channel. Eur J Neurosci. 2008;27(3):605-611.

4. Bautista DM, et al. TRPA1 mediates the inflammatory actions of environmental irritants and proalgesic agents. Cell. 2006;124(6):1269-1282.

5. Trevisani M, et al. 4-Hydroxynonenal, an endogenous aldehyde, causes pain and neurogenic inflammation through activation of the irritant receptor TRPA1. Proc Natl Acad Sci USA. 2007;104(33):13519-13524.

6. Graepel R, Fernandes ES, Aubdool AA, Andersson DA, Bevan S, Brain SD. 4-oxo-2-nonenal (4-ONE): evidence of transient receptor potential ankyrin 1-dependent and -independent nociceptive and vasoactive responses in vivo. J Pharmacol Exp Ther. 2011;337(1):117-124

7. Fernandes ES, Fernandes MA, Keeble JE. The functions of TRPA1 and TRPV1: moving away from sensory nerves. Br J Phar macol. 2012;166(2):510-521.

8. Bertin S, et al. The TRPA1 ion channel is expressed in CD4+ T cells and restrains T-cell-mediated colitis through inhibition of TRPV1. Gut. 2017;66(9):1584-1596.

9. Purhonen AK, Louhivuori LM, Kiehne K, Kerman KE, Herzig KH. TRPA1 channel activation induces cholecystokinin release via extracellular calcium. FEBS Lett. 2008;582(2):229-232.

10. Nozawa K, et al. TRPA1 regulates gastrointestinal motility through serotonin release from enterochromaffin cells. Proc Natl Acad Sci USA. 2009;106(9):3408-3413.

11. Bellono NW, et al. Enterochromaffin cells are gut chemosensors that couple to sensory neural pathways. Cell. 2017;170(1):185-198.e16.

12. Beumer J, et al. Enteroendocrine cells switch hormone expression along the crypt-to-villus BMP signalling gradient. Nat Cell Biol. 2018;20(8):909-916.

13. Gehart $\mathrm{H}$, et al. Identification of enteroendocrine regulators by real-time single-cell differentiation mapping. Cell. 2019;176(5):1158-1173.e16.

14. Brierley SM, et al. The ion channel TRPA1 is required for normal mechanosensation and is modulated by algesic stimuli. Gastroenterology. 2009;137(6):2084-2095.e3.

15. Burakoff R, Percy WH. Studies in vivo and in vitro on effects of PGE2 on colonic motility in rabbits. Am J Physiol. 1992;262(1 Pt 1):G23-G29.

16. Dey I, Lejeune M, Chadee K. Prostaglandin E2 receptor distribution and function in the gastrointestinal tract. Br J Pharmacol. 
2006;149(6):611-623.

17. Iizuka Y, Kuwahara A, Karaki S. Role of PGE2 in the colonic motility: PGE2 generates and enhances spontaneous contractions of longitudinal smooth muscle in the rat colon. J Physiol Sci. 2014;64(2):85-96.

18. Martinez-Cutillas M, Mañé N, Gallego D, Jimenez M, Martin MT. EP2 and EP4 receptors mediate PGE2 induced relaxation in murine colonic circular muscle: pharmacological characterization. Pharmacol Res. 2014;90:76-86.

19. Gershon MD. 5-Hydroxytryptamine (serotonin) in the gastrointestinal tract. Curr Opin Endocrinol Diabetes Obes. 2013;20(1):14-21.

20. Bertrand PP, Bertrand RL. Serotonin release and uptake in the gastrointestinal tract. Auton Neurosci. 2010;153(1-2):47-57.

21. Mawe GM, Hoffman JM. Serotonin signalling in the gut--functions, dysfunctions and therapeutic targets. Nat Rev Gastroenterol Hepatol. 2013;10(8):473-486.

22. Diwakarla S, Fothergill LJ, Fakhry J, Callaghan B, Furness JB. Heterogeneity of enterochromaffin cells within the gastrointestinal tract. Neurogastroenterol Motil. 2017;29(6):null.

23. Martin AM, Lumsden AL, Young RL, Jessup CF, Spencer NJ, Keating DJ. Regional differences in nutrient-induced secretion of gut serotonin. Physiol Rep. 2017;5(6):null.

24. Martin AM, Lumsden AL, Young RL, Jessup CF, Spencer NJ, Keating DJ. The nutrient-sensing repertoires of mouse enterochromaffin cells differ between duodenum and colon. Neurogastroenterol Motil. 2017;29(6):null.

25. Tang EH, Vanhoutte PM. Prostanoids and reactive oxygen species: team players in endothelium-dependent contractions. Pharmacol Ther. 2009;122(2):140-149.

26. Yuhki K, Kashiwagi H, Kojima F, Kawabe J, Ushikubi F. Roles of prostanoids in the pathogenesis of cardiovascular diseases. Int Angiol. 2010;29(2 Suppl):19-27.

27. Kondo T, et al. Prostaglandin E(2) mediates acid-induced heartburn in healthy volunteers. Am J Physiol Gastrointest Liver Physiol. 2013;304(6):G568-G573.

28. Grasa L, Arruebo MP, Plaza MA, Murillo MD. PGE(2) receptors and their intracellular mechanisms in rabbit small intestine. Prostaglandins Other Lipid Mediat. 2006;79(3-4):206-217.

29. Poole DP, et al. Transient receptor potential ankyrin 1 is expressed by inhibitory motoneurons of the mouse intestine. Gastroenterology. 2011;141(2):565-575.

30. Raisinghani M, et al. Activation characteristics of transient receptor potential ankyrin 1 and its role in nociception. Am J Physiol, Cell Physiol. 2011;301(3):C587-C600.

31. Maruyama T, et al. Influence of local treatments with capsaicin or allyl isothiocyanate in the sensitization phase of a fluorescein-isothiocyanate-induced contact sensitivity model. Int Arch Allergy Immunol. 2007;143(2):144-154

32. Powell DW, Pinchuk IV, Saada JI, Chen X, Mifflin RC. Mesenchymal cells of the intestinal lamina propria. Annu Rev Physiol. 2011;73:213-237.

33. Pinchuk IV, Mifflin RC, Saada JI, Powell DW. Intestinal mesenchymal cells. Curr Gastroenterol Rep. 2010;12(5):310-318

34. Roulis M, Flavell RA. Fibroblasts and myofibroblasts of the intestinal lamina propria in physiology and disease. Differentiation. 2016;92(3):116-131.

35. Kurahara LH, et al. Activation of myofibroblast TRPA1 by steroids and pirfenidone ameliorates fibrosis in experimental Crohn's disease. Cell Mol Gastroenterol Hepatol. 2018;5(3):299-318.

36. Watsky MA, Weber KT, Sun Y, Postlethwaite A. New insights into the mechanism of fibroblast to myofibroblast transformation and associated pathologies. Int Rev Cell Mol Biol. 2010;282:165-192.

37. Sarna SK. Colonic motility: From bench side to bedside. San Rafael (CA); 2010

38. Chassaing B, Aitken JD, Malleshappa M, Vijay-Kumar M. Dextran sulfate sodium (DSS)-induced colitis in mice. Curr Protoc Immunol. 2014;104:Unit 15.25.

39. Wirtz S, Neufert C, Weigmann B, Neurath MF. Chemically induced mouse models of intestinal inflammation. Nat Protoc. 2007;2(3):541-546.

40. Okayasu I, Hatakeyama S, Yamada M, Ohkusa T, Inagaki Y, Nakaya R. A novel method in the induction of reliable experimental acute and chronic ulcerative colitis in mice. Gastroenterology. 1990;98(3):694-702.

41. Li G, et al. Oral pirfenidone protects against fibrosis by inhibiting fibroblast proliferation and TGF- $\beta$ signaling in a murine colitis model. Biochem Pharmacol. 2016;117:57-67.

42. Scheibe K, et al. IL-36R signalling activates intestinal epithelial cells and fibroblasts and promotes mucosal healing in vivo. Gut. 2017;66(5):823-838.

43. Engel MA, et al. TRPA1 and substance P mediate colitis in mice. Gastroenterology. 2011;141(4):1346-1358.

44. Farombi EO, et al. Dietary protocatechuic acid ameliorates dextran sulphate sodium-induced ulcerative colitis and hepatotoxicity in rats. Food Funct. 2016;7(2):913-921.

45. Kogure $\mathrm{Y}$, et al. Elevated $\mathrm{H}_{2} \mathrm{O}_{2}$ levels in trinitrobenzene sulfate-induced colitis rats contributes to visceral hyperalgesia through interaction with the transient receptor potential ankyrin 1 cation channel. J Gastroenterol Hepatol. 2016;31(6):1147-1153.

46. Kobayashi K, et al. Distinct expression of TRPM8, TRPA1, and TRPV1 mRNAs in rat primary afferent neurons with adelta/c-fibers and colocalization with trk receptors. J Comp Neurol. 2005;493(4):596-606.

47. Wang S, et al. Negative regulation of TRPA1 by AMPK in primary sensory neurons as a potential mechanism of painful diabetic neuropathy. Diabetes. 2018;67(1):98-109. 